\title{
Bis(phosphine)hydridorhodacarborane Derivatives of 1,1'-Bis(ortho- carborane) and their Catalysis of Alkene Isomerization and the Hydrosilylation of Acetophenone
}

Antony P. Y. Chan, John A. Parkinson, Georgina M. Rosair and Alan J. Welch

Institute of Chemical Sciences, Heriot-Watt University, Edinburgh EH14 4AS, U.K. and

Department of Pure and Applied Chemistry, University of Strathclyde, Glasgow G1 1XL, U.K.

\section{SUPPORTING INFORMATION}

Part A: Crystallographic data.

Part B1: ${ }^{1} \mathrm{H},{ }^{11} \mathrm{~B}\left\{{ }^{1} \mathrm{H}\right\}$ and ${ }^{31} \mathrm{P}\left\{{ }^{1} \mathrm{H}\right\}$ NMR spectra of all new compounds.

Part B2: ${ }^{103}$ Rh NMR study of compounds I and V.

Part C: Full details of catalytic studies. 


\begin{tabular}{|c|c|c|c|c|c|c|}
\hline & $\mathbf{1} \cdot 0.5 \mathrm{CH}_{2} \mathrm{Cl}_{2}$ & $2 \alpha \cdot 2 \mathrm{CH}_{2} \mathrm{Cl}_{2}$ & $2 \beta \cdot 1.5 \mathrm{CH}_{2} \mathrm{Cl}_{2}$ & $3 \alpha \cdot 2.5 \mathrm{CH}_{2} \mathrm{Cl}_{2}$ & $3 \beta \cdot 2.5 \mathrm{CH}_{2} \mathrm{Cl}_{2}$ & V \\
\hline $\mathrm{CCDC}$ & 1964963 & 1964964 & 1964965 & 1964966 & 1964967 & 1964968 \\
\hline Formula & $\mathrm{C}_{40.5} \mathrm{H}_{53} \mathrm{~B}_{19} \mathrm{ClP}_{2} \mathrm{Rh}$ & $\mathrm{C}_{52} \mathrm{H}_{69} \mathrm{~B}_{18} \mathrm{Cl}_{4} \mathrm{P}_{2} \mathrm{RhRu}$ & $\mathrm{C}_{51.5} \mathrm{H}_{68} \mathrm{~B}_{18} \mathrm{Cl}_{3} \mathrm{P}_{2} \mathrm{RhRu}$ & $\mathrm{C}_{52.5} \mathrm{H}_{71} \mathrm{~B}_{18} \mathrm{Cl}_{5} \mathrm{CoP}_{2} \mathrm{Rh}$ & $\mathrm{C}_{52.5} \mathrm{H}_{71} \mathrm{~B}_{18} \mathrm{Cl}_{5} \mathrm{CoP}_{2} \mathrm{Rh}$ & $\mathrm{C}_{38} \mathrm{H}_{42} \mathrm{~B}_{9} \mathrm{P}_{2} \mathrm{Rh}$ \\
\hline$M$ & 945.52 & 1296.37 & 1253.90 & 1297.70 & 1297.70 & 760.85 \\
\hline Crystal system & Monoclinic & Monoclinic & Triclinic & Monoclinic & Monoclinic & Monoclinic \\
\hline Space group & $P 2{ }_{1} / c$ & $P 2_{1} / c$ & $P \overline{1}$ & $P 2{ }_{1} / c$ & $P 2{ }_{1} / c$ & $P 2_{1} / n$ \\
\hline$a / \AA$ & $14.3180(4)$ & $24.3210(5)$ & $10.7800(2)$ & $20.848(10)$ & $21.082(4)$ & $12.1833(4)$ \\
\hline$b / \AA$ & $24.5697(6)$ & $14.1399(2)$ & $14.7172(3)$ & $29.306(13)$ & $29.554(6)$ & $15.9589(5)$ \\
\hline$c / \AA$ & $13.0131(4)$ & $18.6151(3)$ & $18.7587(3)$ & $20.172(9)$ & $20.354(4)$ & $18.9662(7)$ \\
\hline$\alpha /^{\circ}$ & 90 & 90 & $90.2185(16)$ & 90 & 90 & 90 \\
\hline$\beta /^{\circ}$ & $94.727(3)$ & $107.293(2)$ & $106.5492(18)$ & $97.299(12)$ & $97.60(3)$ & $90.4340(10)$ \\
\hline$\gamma /{ }^{\circ}$ & 90 & 90 & $101.0331(15)$ & 90 & 90 & 90 \\
\hline$U / \AA^{3}$ & $4562.3(2)$ & $6112.32(18)$ & $2794.68(10)$ & $12225(10)$ & $12571(4)$ & $3687.5(2)$ \\
\hline$Z, Z^{\prime}$ & 4,1 & 4,1 & 2,1 & 8,2 & 8,2 & 4,1 \\
\hline$F(000) / \mathrm{e}$ & 1932.0 & 2632.0 & 1274 & 5304.0 & 5304.0 & 1560.0 \\
\hline$D_{\text {calc }} / \mathrm{Mg} \mathrm{m}^{-3}$ & 1.377 & 1.409 & 1.490 & 1.410 & 1.371 & 1.370 \\
\hline$X$-radiation & Мo- $K_{\alpha}$ & $\mathrm{Cu}-K_{\alpha}$ & $\mathrm{Cu}-K_{\alpha}$ & Мо- $K_{\alpha}$ & Мо- $K_{\alpha}$ & Мo- $K_{\alpha}$ \\
\hline$\lambda / \AA$ & 0.71073 & 1.54178 & 1.54178 & 0.71073 & 0.71073 & 0.71073 \\
\hline$\mu / \mathrm{mm}^{-1}$ & 0.536 & 6.540 & 6.706 & 0.849 & 0.826 & 0.578 \\
\hline$\theta_{\max } /^{\circ}$ & 27.48 & 68.23 & 68.24 & 26.29 & 24.83 & 28.30 \\
\hline Data measured & 38649 & 48915 & 51318 & 174815 & 117405 & 42882 \\
\hline Unique data & 10245 & 11100 & 10174 & 24342 & 20919 & 9122 \\
\hline$R_{\text {int }}$ & 0.0725 & 0.0690 & 0.0711 & 0.2373 & 0.3237 & 0.0668 \\
\hline$R, \mathrm{w} R_{2}$ (obs. data) & $0.0635,0.1373$ & $0.0605,0.1651$ & $0.0513,0.1418$ & $0.1462,0.3628$ & $0.1091,0.2503$ & $0.0323,0.0778$ \\
\hline$S$ & 1.050 & 1.065 & 1.039 & 1.307 & 0.955 & 1.052 \\
\hline Variables & 625 & 742 & 716 & 1340 & 1340 & 487 \\
\hline$E_{\max }, E_{\min } / \mathrm{e} \AA^{-3}$ & $2.52,-0.99$ & $1.50,-1.23$ & $2.37,-1.01$ & $6.69^{a},-2.50$ & $1.54,-2.63$ & $0.52,-0.55$ \\
\hline
\end{tabular}

${ }^{a}$ residual electron density ca. 4 and $6 \mathrm{e}^{-3}$ close to metal atoms in both independent molecules 


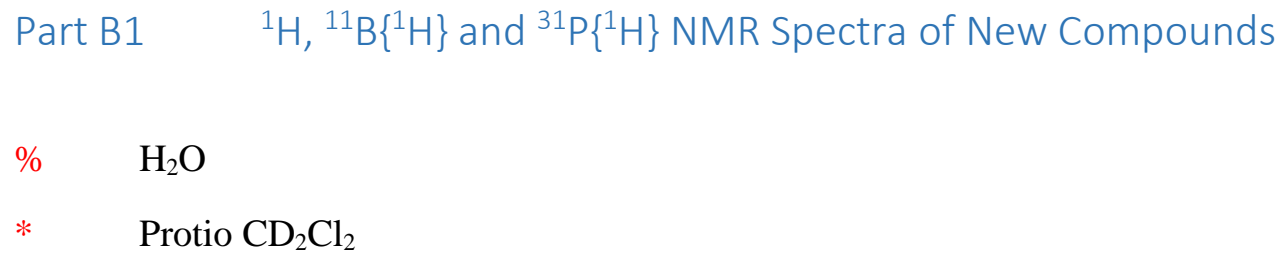

Compound 1: [8-(1'-closo-1',2'- $\left.\mathrm{C}_{2} \mathrm{~B}_{10} \mathrm{H}_{11}\right)-2-\mathrm{H}-2,2-\left(\mathrm{PPh}_{3}\right)_{2}$-closo-2,1,8- $\left.\mathrm{RhC}_{2} \mathrm{~B}_{9} \mathrm{H}_{10}\right]$ ${ }^{1} \mathrm{H}$ NMR $\left(\mathrm{CD}_{2} \mathrm{Cl}_{2}\right)$ :

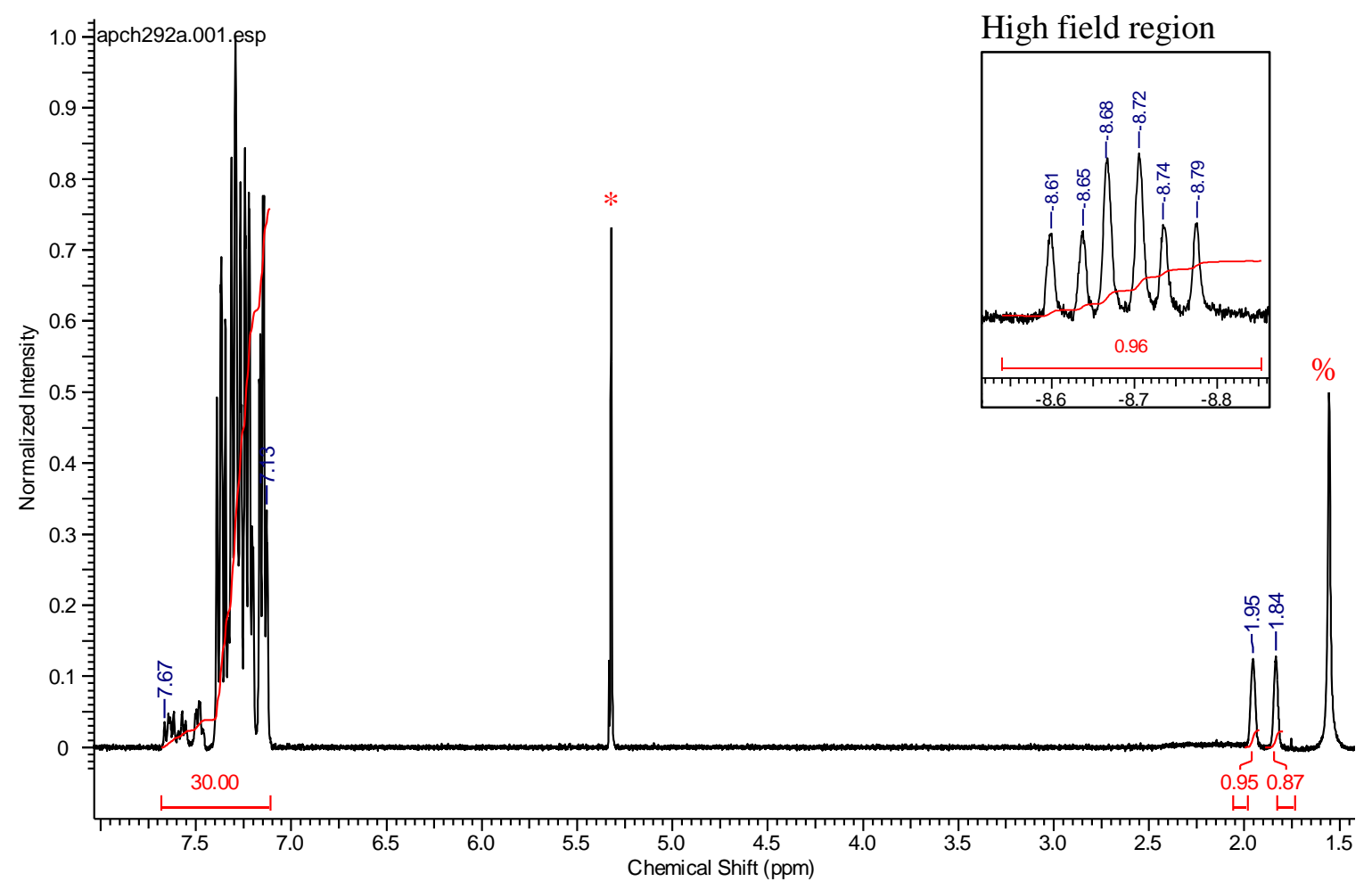


${ }^{11} \mathrm{~B}\left\{{ }^{1} \mathrm{H}\right\}$ NMR $\left(\mathrm{CD}_{2} \mathrm{Cl}_{2}\right)$ :

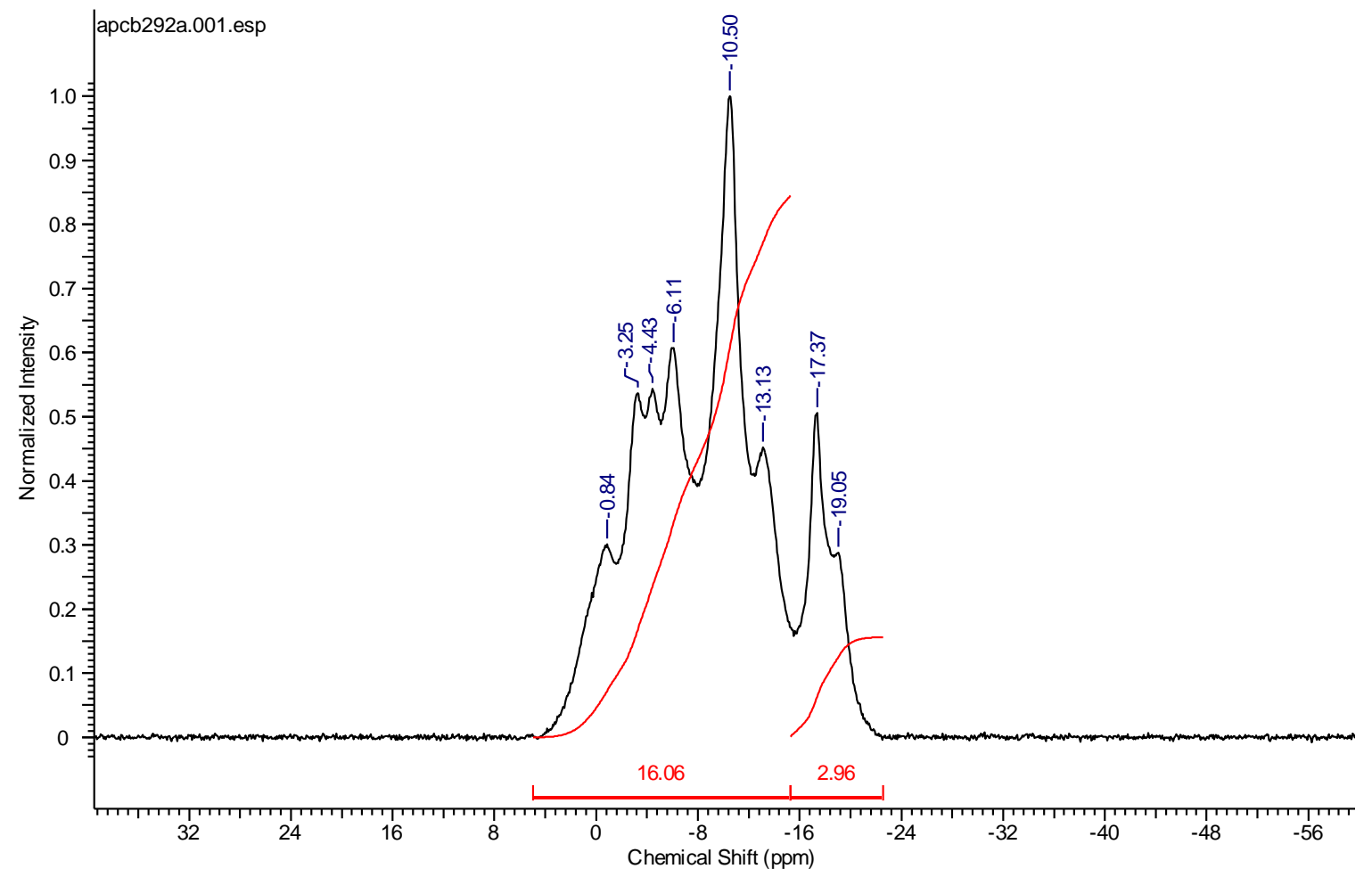

${ }^{31} \mathrm{P}\left\{{ }^{1} \mathrm{H}\right\}$ NMR $\left(\mathrm{CD}_{2} \mathrm{Cl}_{2}\right)$ :

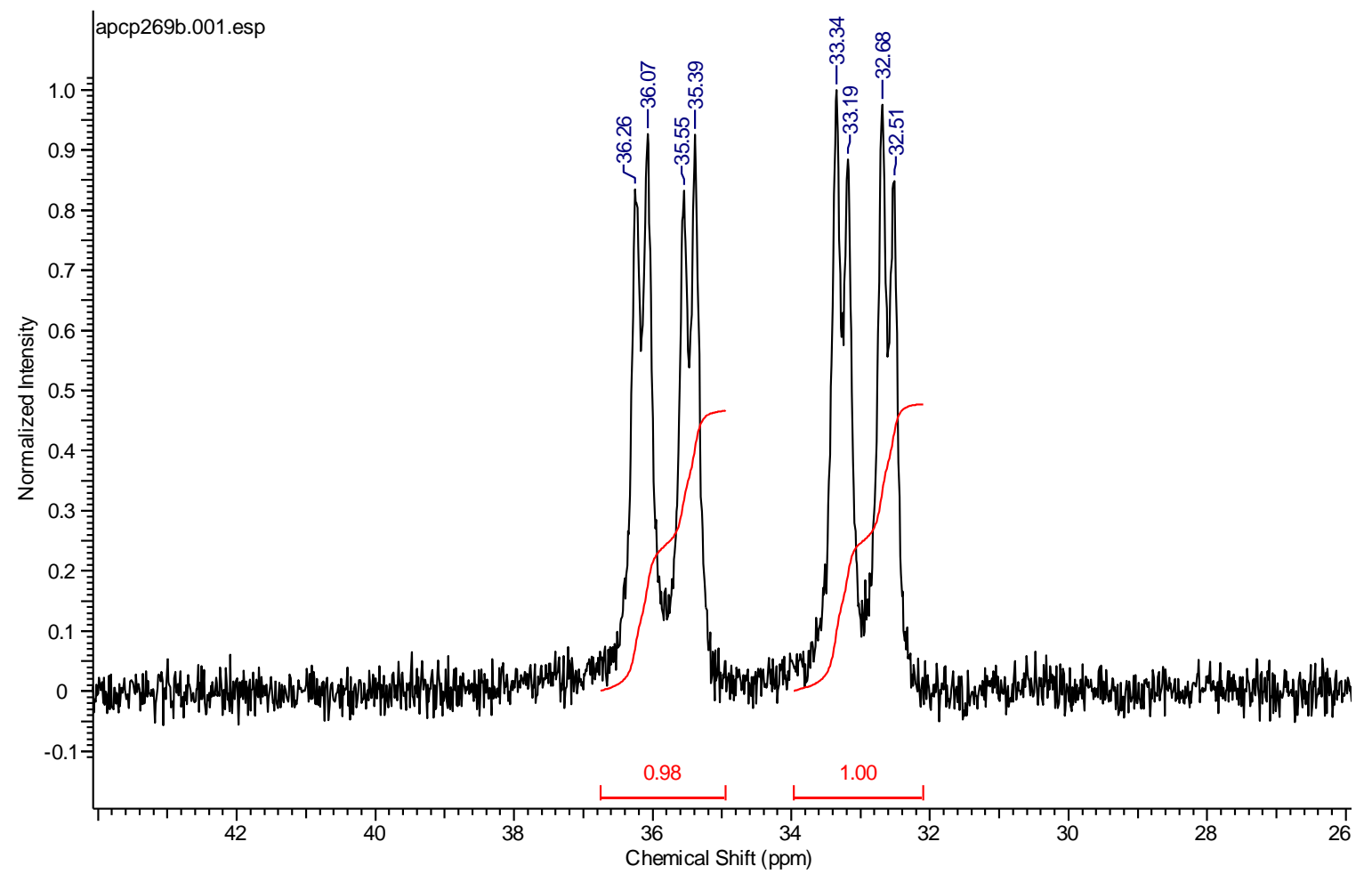


Compound 2 $\alpha$ : $\alpha$-[8-(8'-2'-(p-cymene)-closo-2', 1', $\left.8^{\prime}-\mathrm{RuC}_{2} \mathrm{~B}_{9} \mathrm{H}_{10}\right)-2-\mathrm{H}-2,2-\left(\mathrm{PPh}_{3}\right)_{2}$-closo-2, 1,8$\mathrm{RhC}_{2} \mathrm{~B}_{9} \mathrm{H}_{10}$ ]

${ }^{1} \mathrm{H}$ NMR $\left(\mathrm{CD}_{2} \mathrm{Cl}_{2}\right)$ :

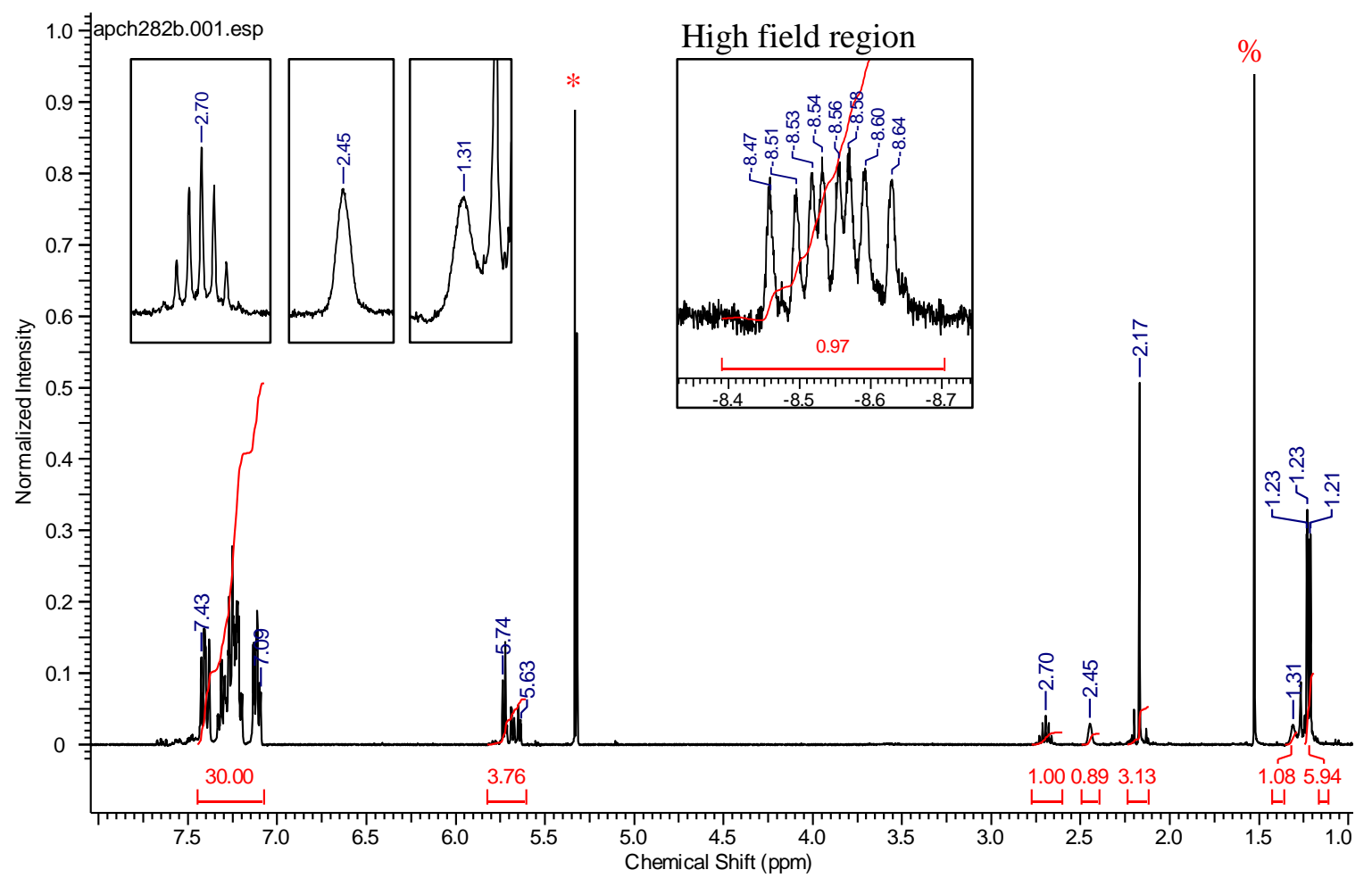

${ }^{11} \mathrm{~B}\left\{{ }^{1} \mathrm{H}\right\}$ NMR $\left(\mathrm{CD}_{2} \mathrm{Cl}_{2}\right)$ :

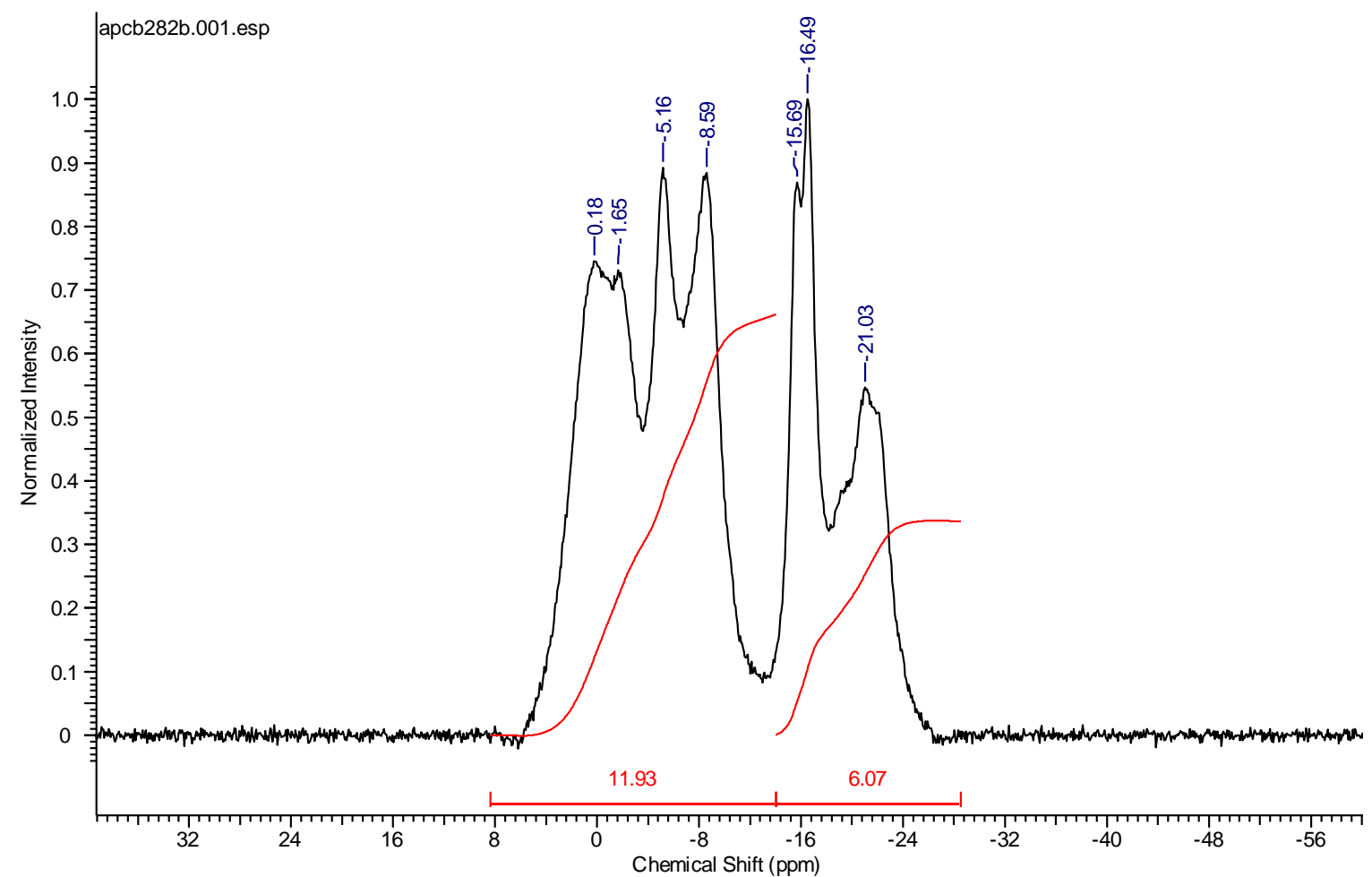




\section{${ }^{31} \mathrm{P}\left\{{ }^{1} \mathrm{H}\right\}$ NMR $\left(\mathrm{CD}_{2} \mathrm{Cl}_{2}\right)$ :}

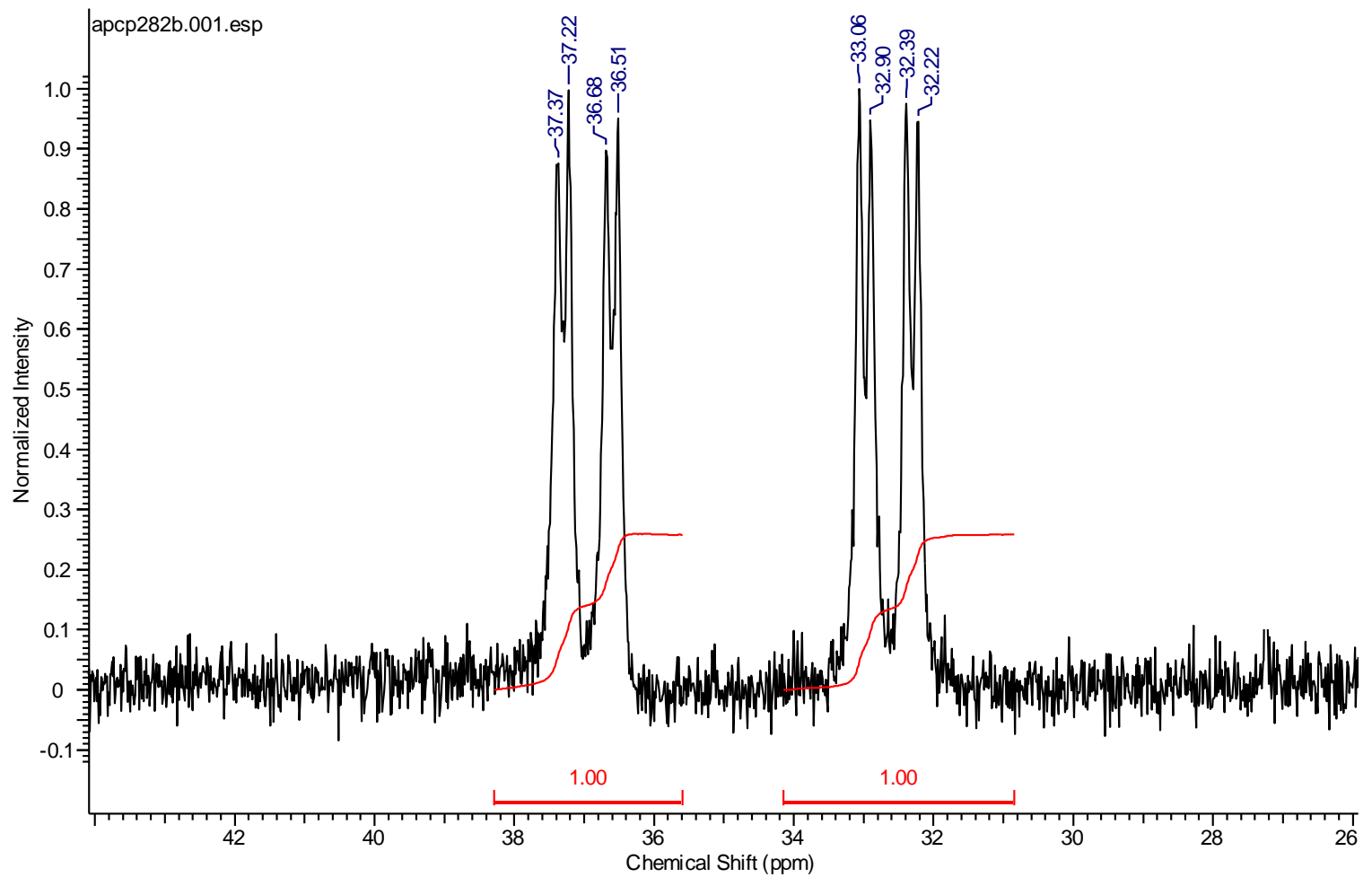




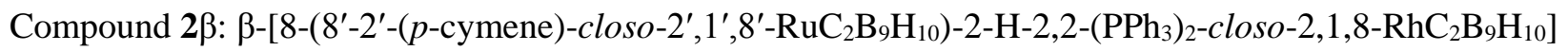
${ }^{1} \mathrm{H}$ NMR $\left(\mathrm{CD}_{2} \mathrm{Cl}_{2}\right)$ :

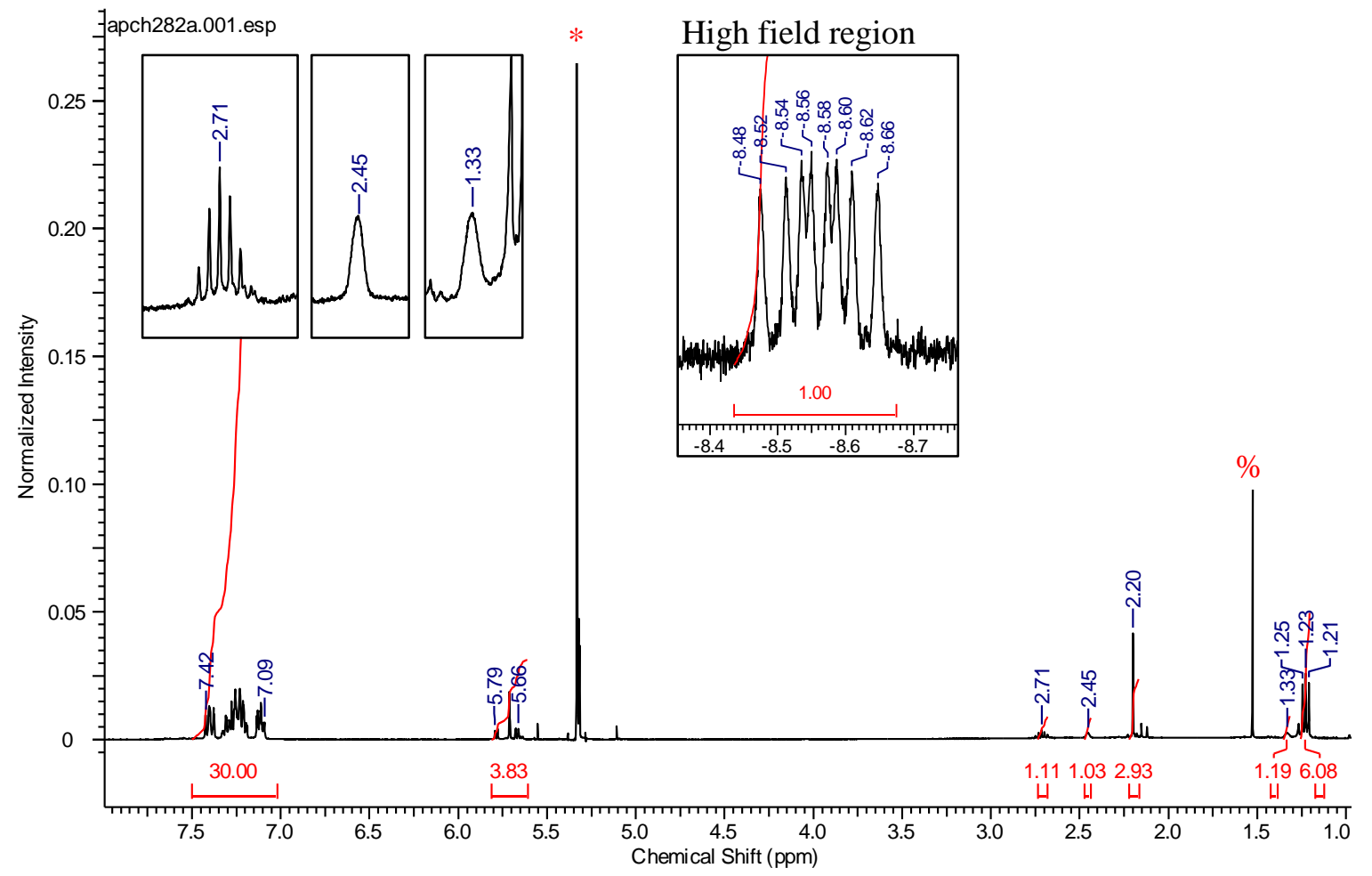

${ }^{11} \mathrm{~B}\left\{{ }^{1} \mathrm{H}\right\}$ NMR $\left(\mathrm{CD}_{2} \mathrm{Cl}_{2}\right)$ :

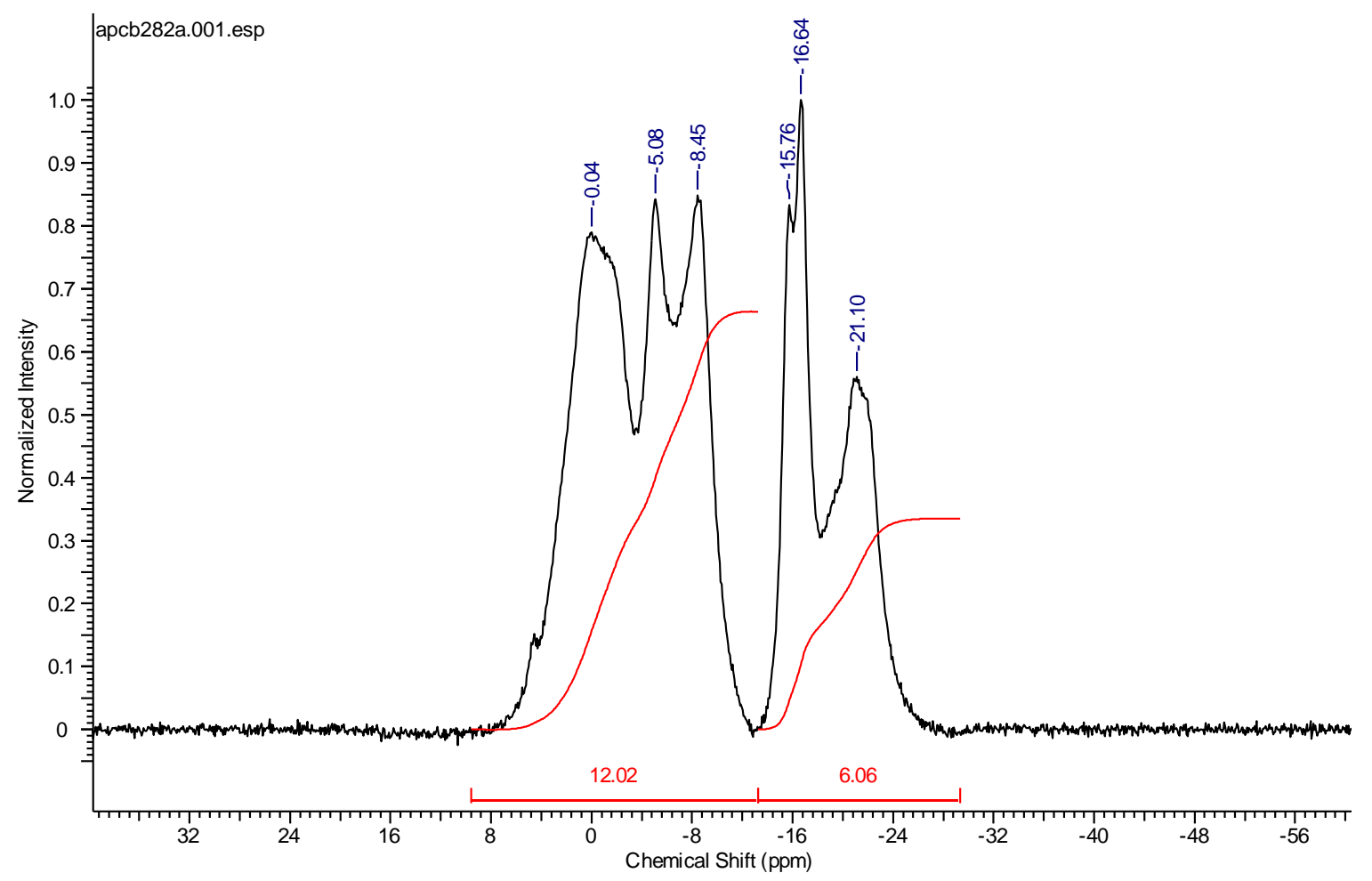


${ }^{31} \mathrm{P}\left\{{ }^{1} \mathrm{H}\right\}$ NMR $\left(\mathrm{CD}_{2} \mathrm{Cl}_{2}\right)$ :

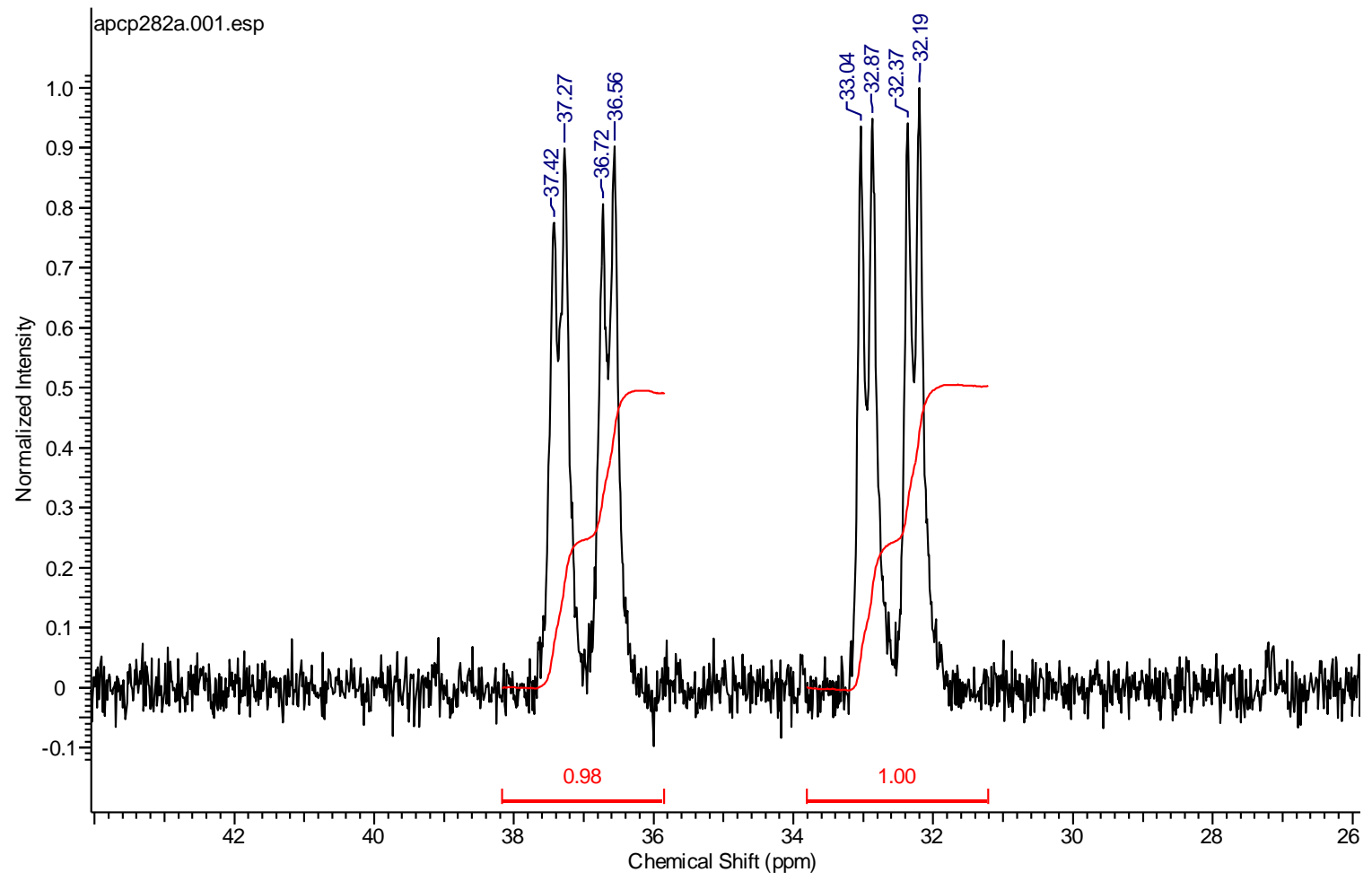

8 
Compound 3 $\alpha$ : $\alpha$-[8-(8'-2'-Cp*-closo-2', 1', $\left.8^{\prime}-\mathrm{CoC}_{2} \mathrm{~B}_{9} \mathrm{H}_{10}\right)-2-\mathrm{H}-2,2-\left(\mathrm{PPh}_{3}\right)_{2}-$ closo-2, 1,8- $\left.\mathrm{RhC}_{2} \mathrm{~B}_{9} \mathrm{H}_{10}\right]$ ${ }^{1} \mathrm{H}$ NMR $\left(\mathrm{CD}_{2} \mathrm{Cl}_{2}\right)$ :

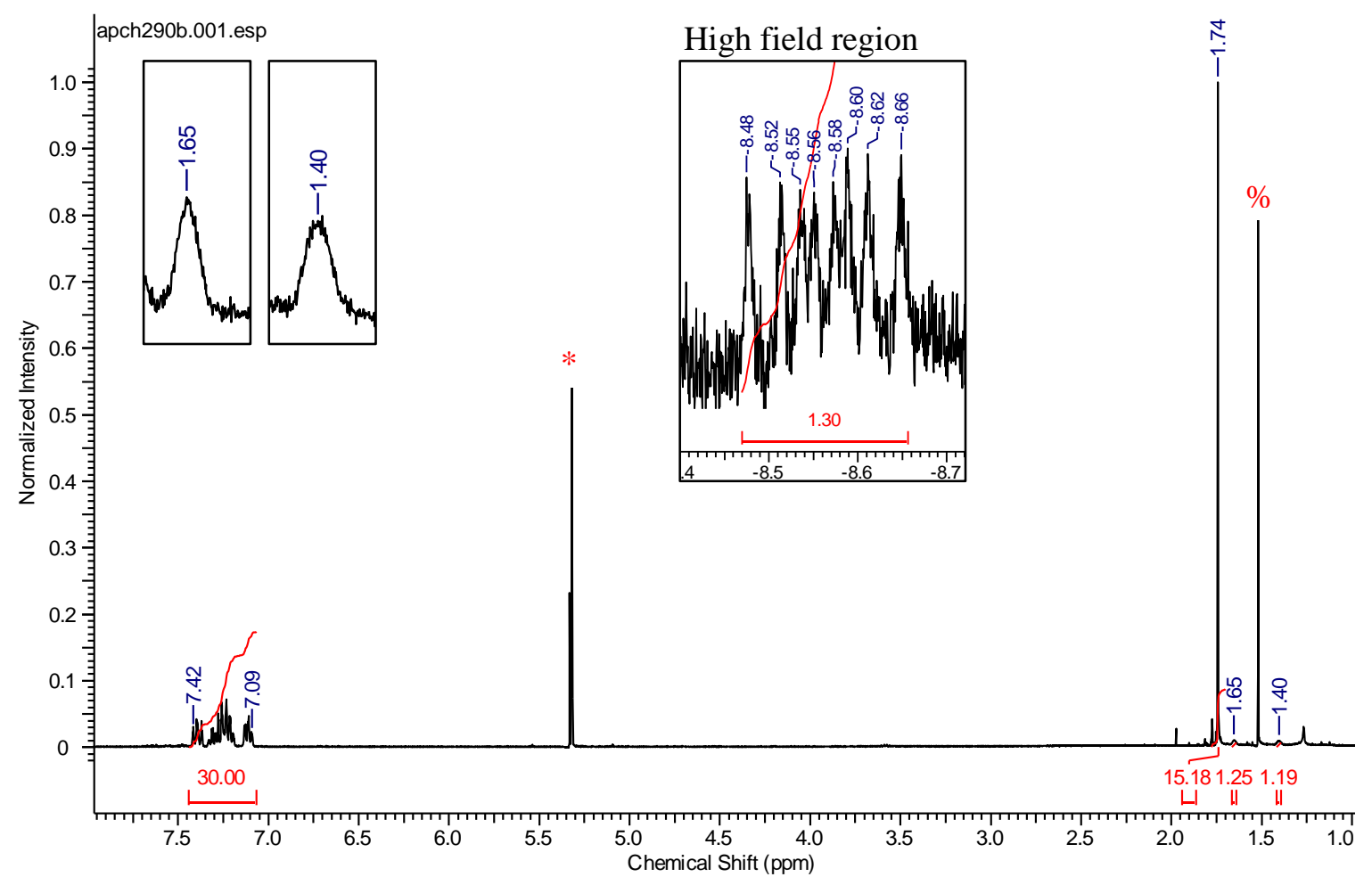

${ }^{11} \mathrm{~B}\left\{{ }^{1} \mathrm{H}\right\}$ NMR $\left(\mathrm{CD}_{2} \mathrm{Cl}_{2}\right)$ :

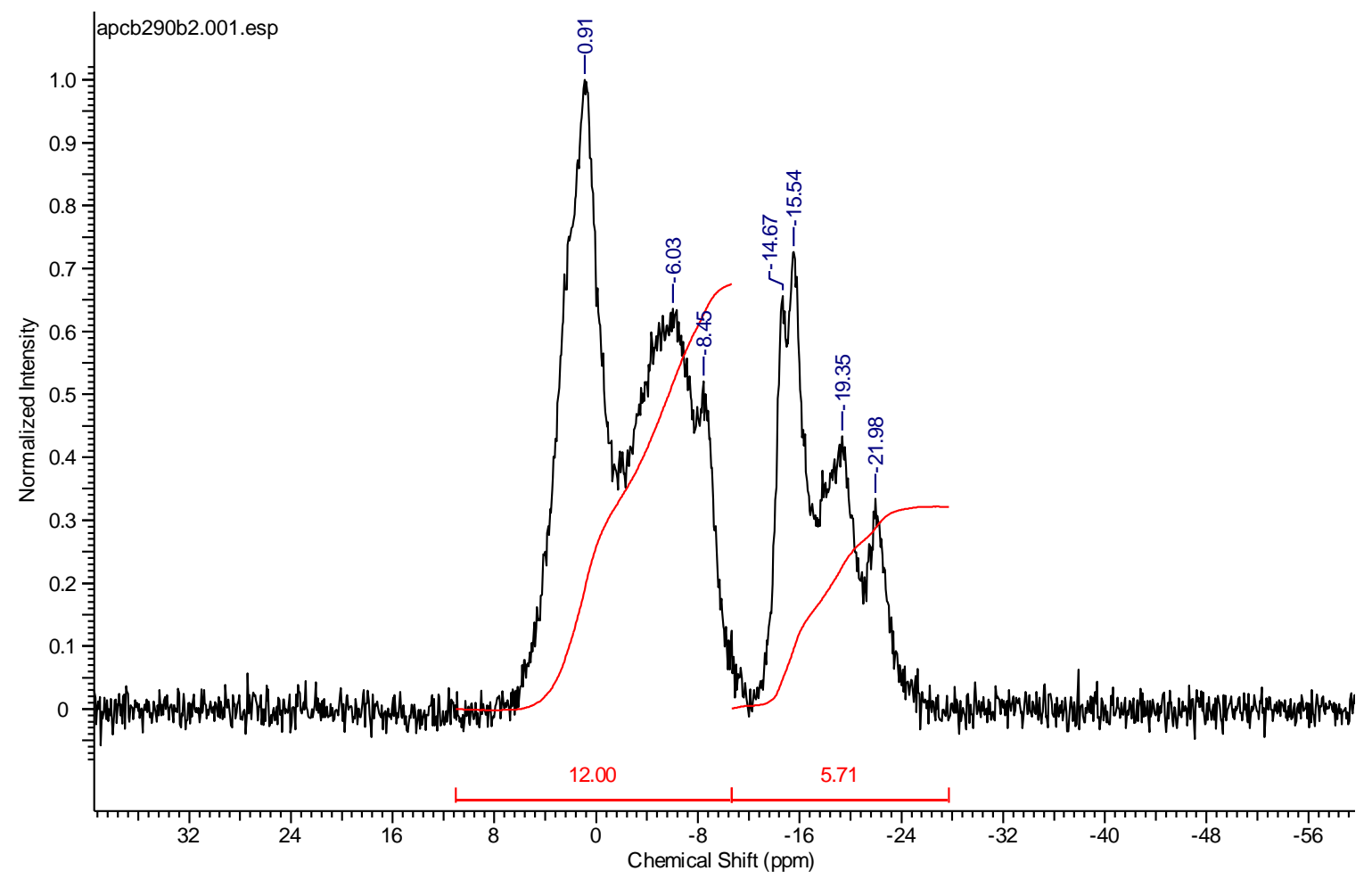


${ }^{31} \mathrm{P}\left\{{ }^{1} \mathrm{H}\right\}$ NMR $\left(\mathrm{CD}_{2} \mathrm{Cl}_{2}\right)$ :

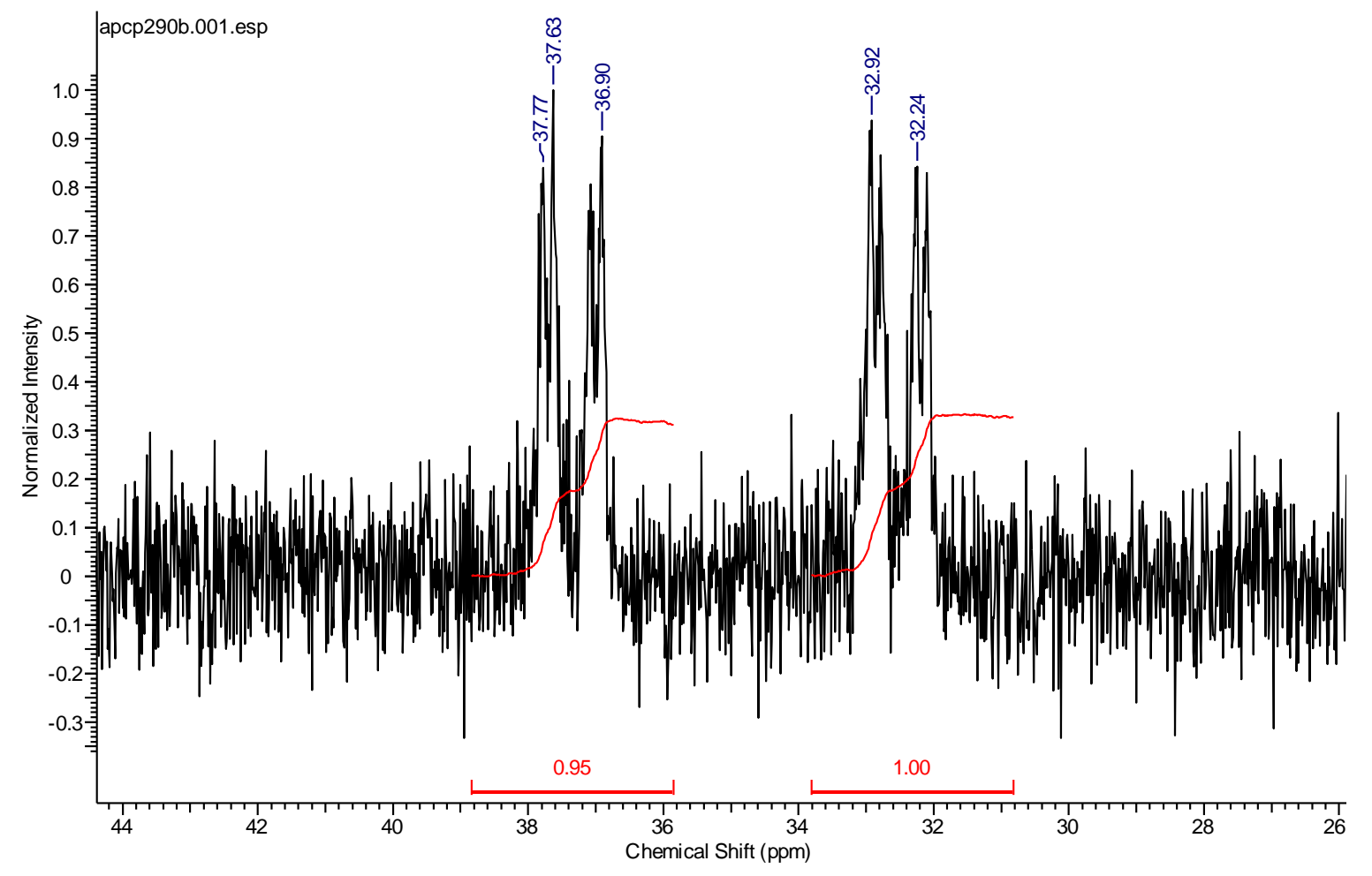




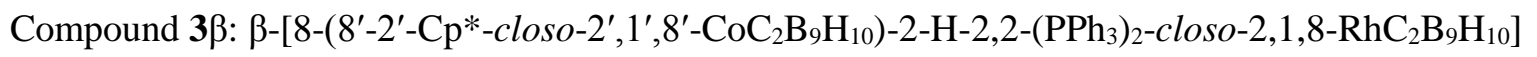
${ }^{1} \mathrm{H}$ NMR $\left(\mathrm{CD}_{2} \mathrm{Cl}_{2}\right)$ :

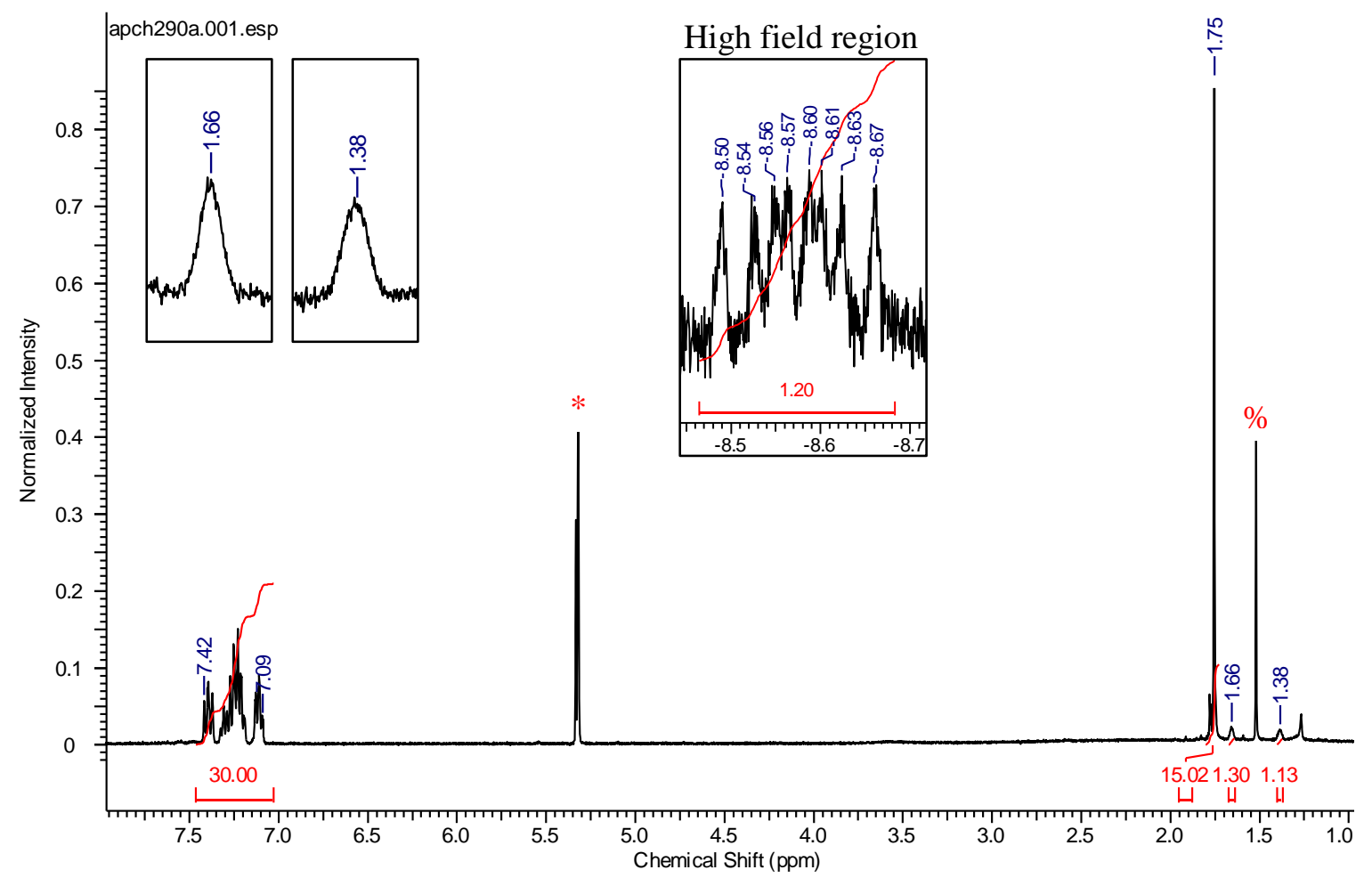

${ }^{11} \mathrm{~B}\left\{{ }^{1} \mathrm{H}\right\}$ NMR $\left(\mathrm{CD}_{2} \mathrm{Cl}_{2}\right)$ :

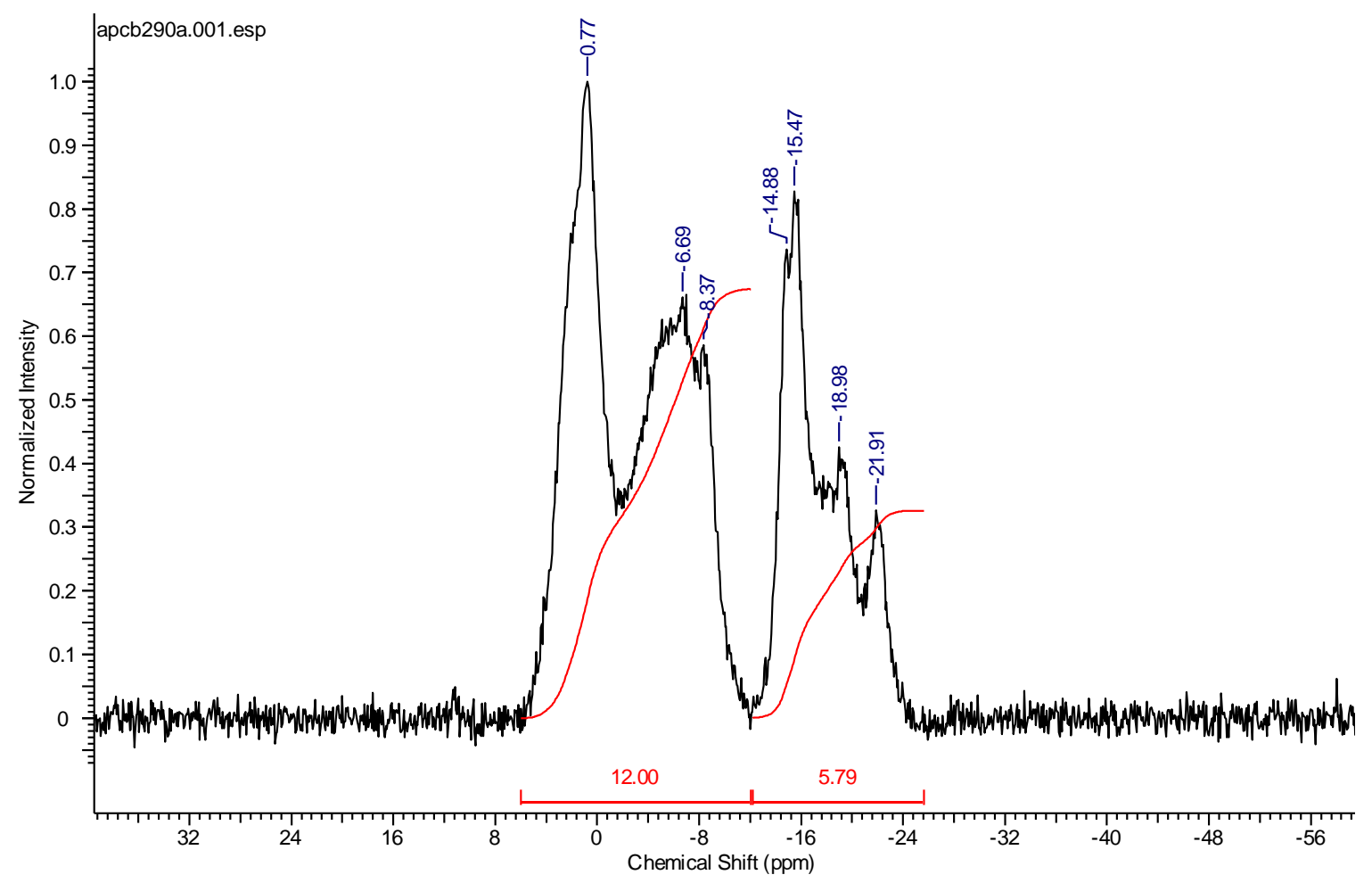


${ }^{31} \mathrm{P}\left\{{ }^{1} \mathrm{H}\right\}$ NMR $\left(\mathrm{CD}_{2} \mathrm{Cl}_{2}\right)$ :

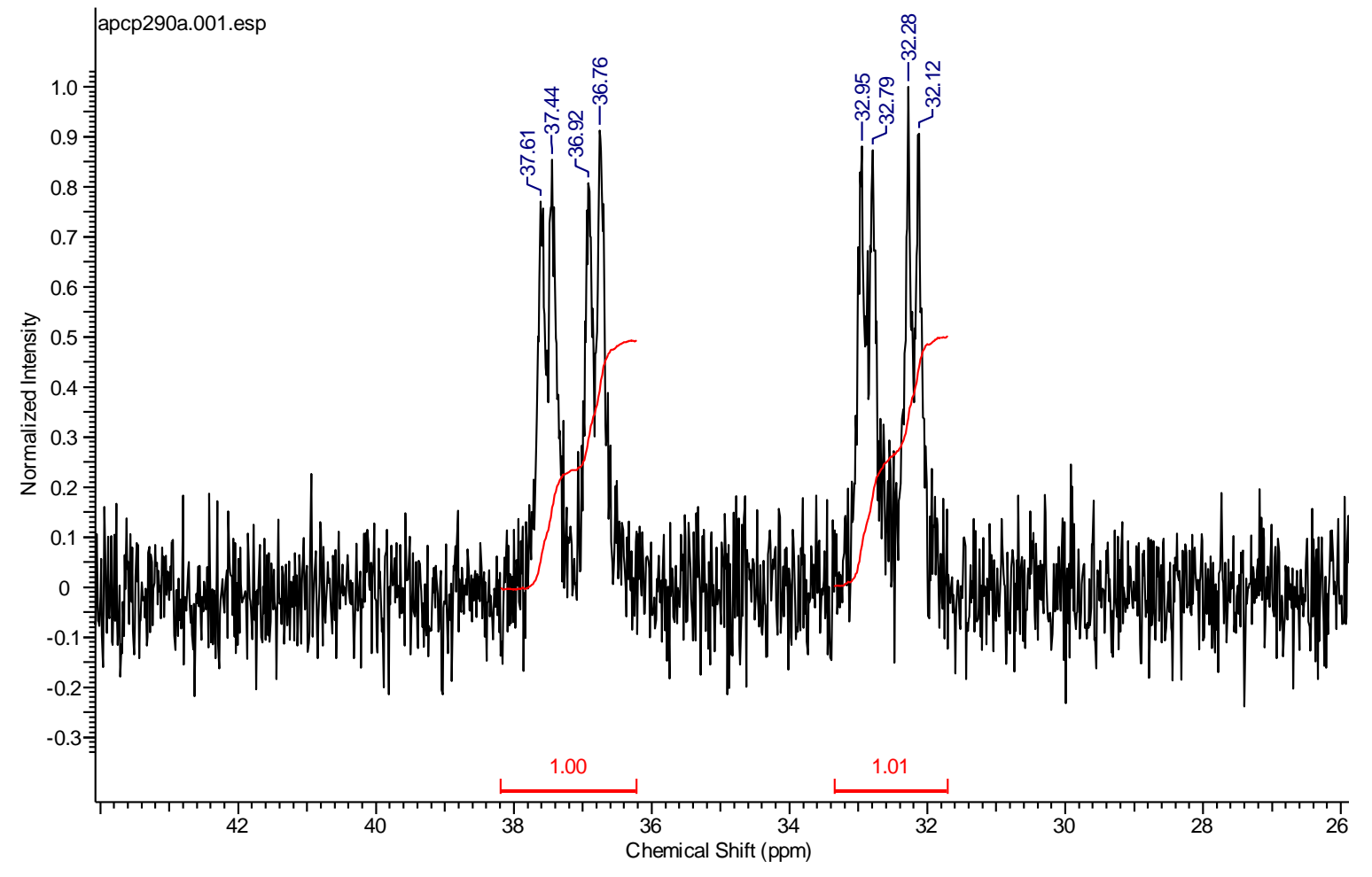


Compound V: [2-H-2,2-( $\left.\mathrm{PPh}_{3}\right)_{2}$-closo-2,1,12- $\left.\mathrm{RhC}_{2} \mathrm{~B}_{9} \mathrm{H}_{11}\right]$

${ }^{1} \mathrm{H}$ NMR $\left(\mathrm{CD}_{2} \mathrm{Cl}_{2}\right)$ :

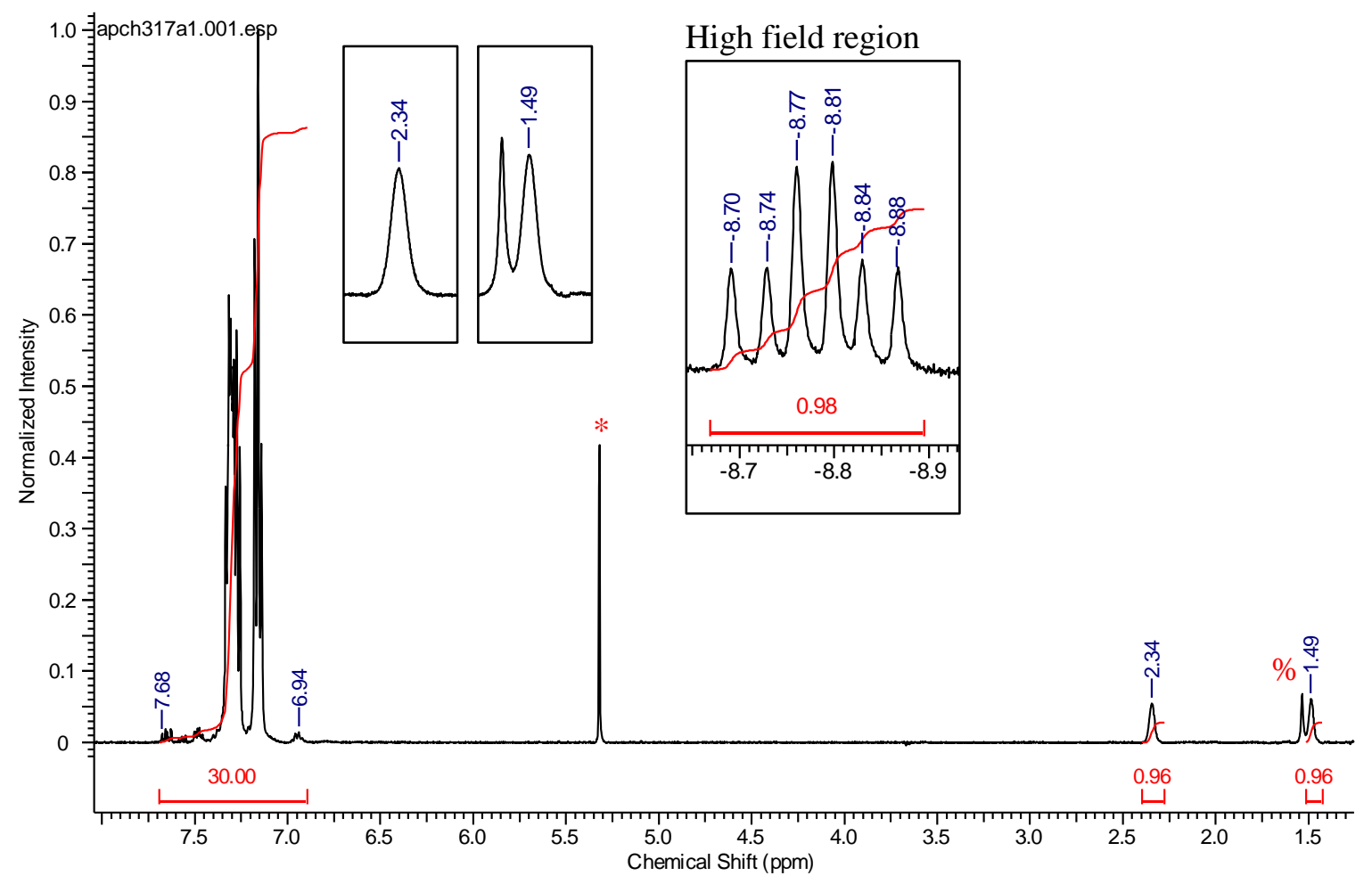

${ }^{11} \mathrm{~B}\left\{{ }^{1} \mathrm{H}\right\}$ NMR $\left(\mathrm{CD}_{2} \mathrm{Cl}_{2}\right)$ :

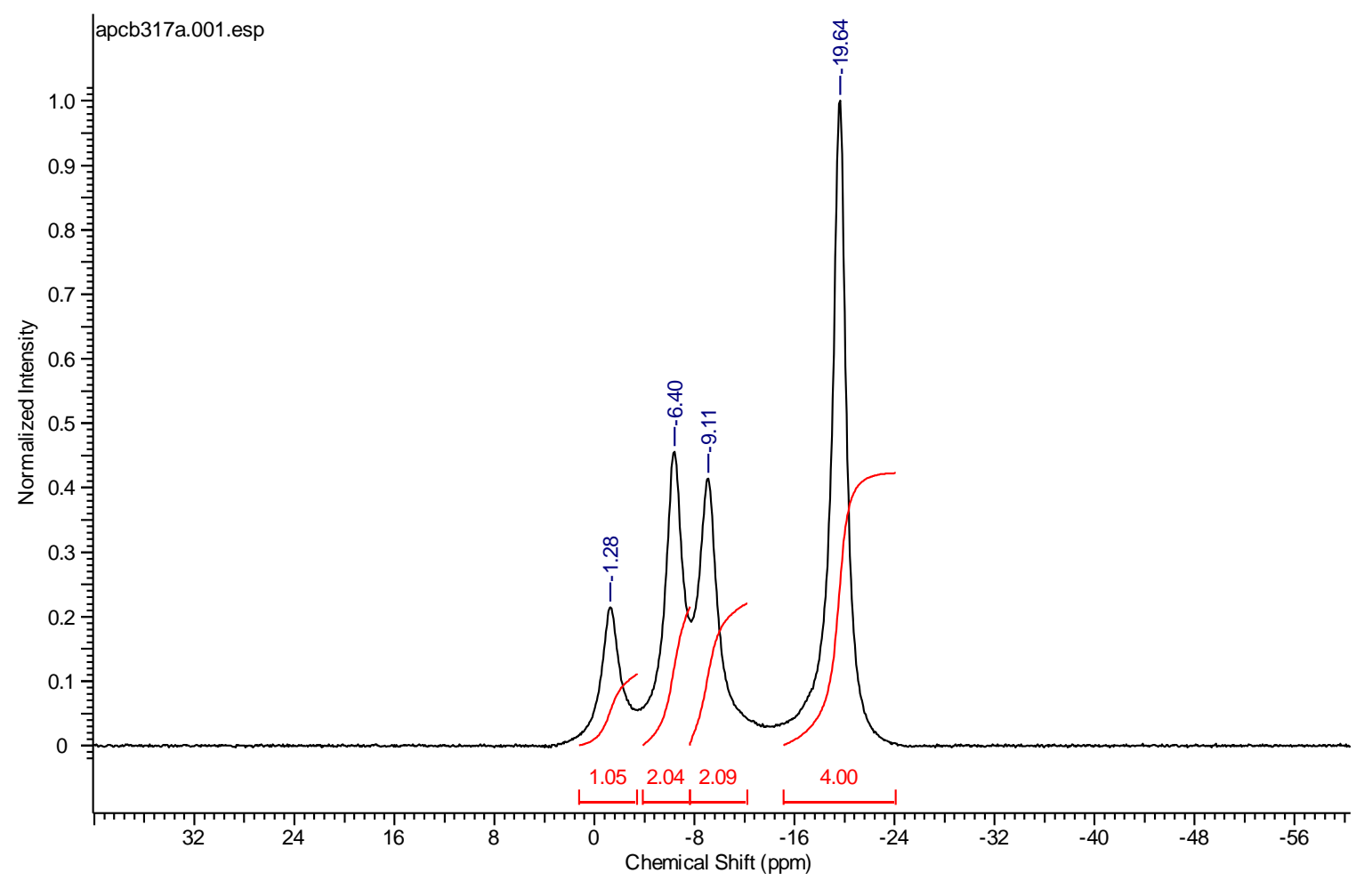




\section{${ }^{31} \mathrm{P}\left\{{ }^{1} \mathrm{H}\right\}$ NMR $\left(\mathrm{CD}_{2} \mathrm{Cl}_{2}\right)$ :}

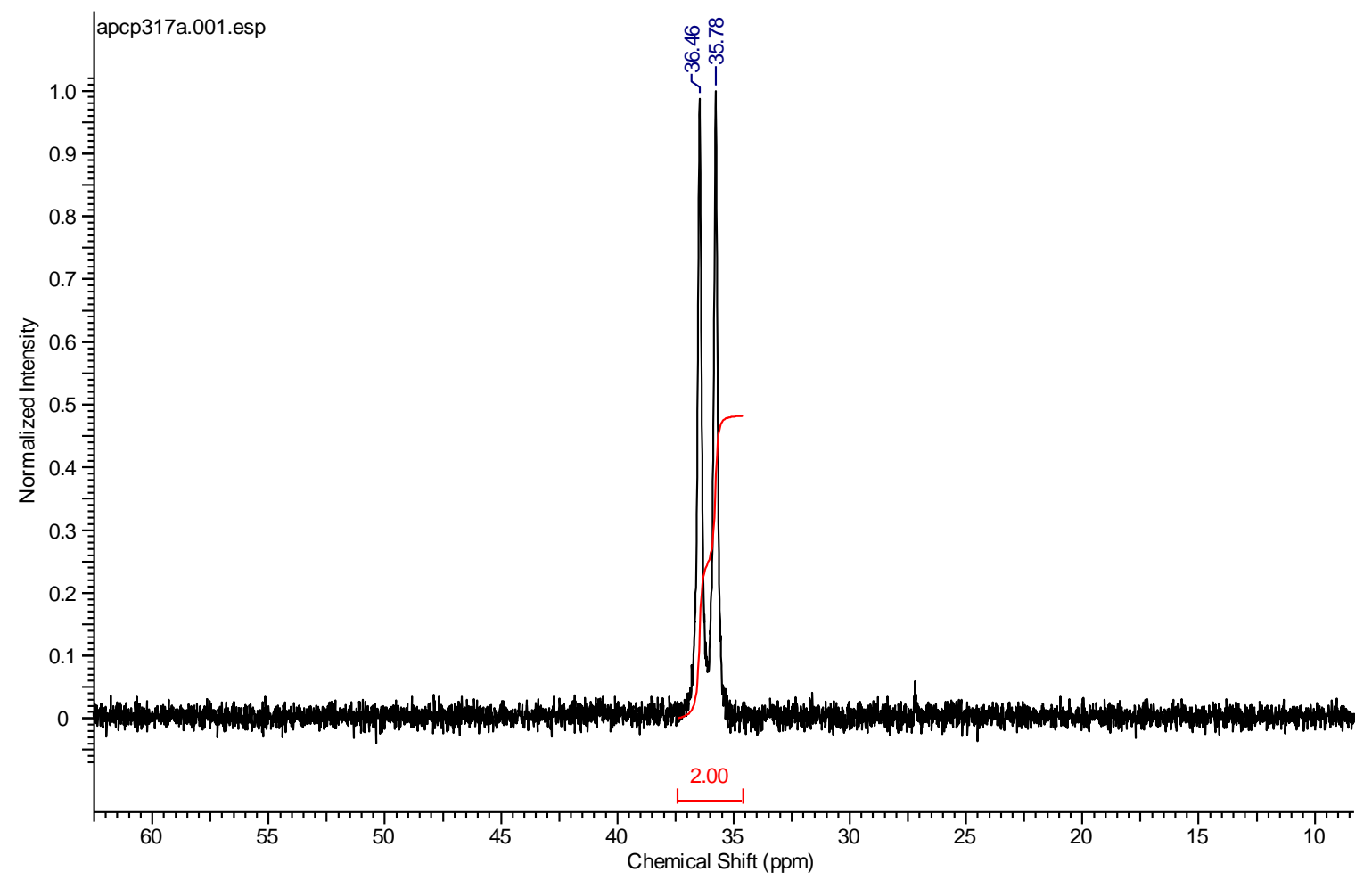




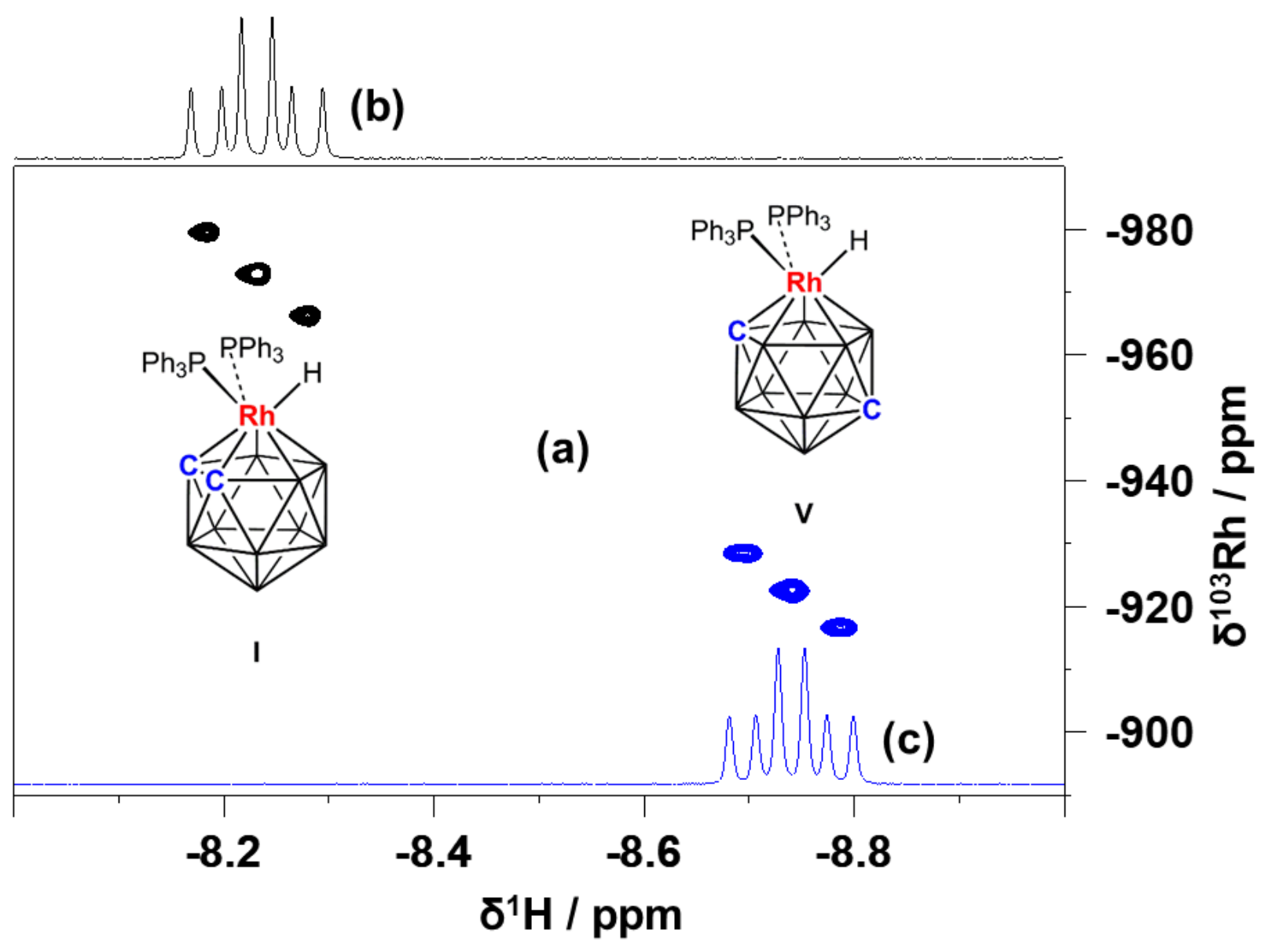

Figure S1. ${ }^{1} \mathrm{H},{ }^{103} \mathrm{Rh}$ correlation data for rhodacarborane clusters $\mathbf{I}$ and $\mathbf{V}$ acquired at a magnetic field of 14.1 T (600.130 MHz for proton resonance). (a) Overlay of the $2 \mathrm{D}\left[{ }^{1} \mathrm{H},{ }^{103} \mathrm{Rh}\right] \mathrm{HMQC}$ NMR spectra for I (black contours) and $\mathbf{V}$ (blue contours) at their respective ${ }^{1} \mathrm{H}$ and ${ }^{103} \mathrm{Rh}$ chemical shifts. In each case the passive ${ }^{1} \mathrm{~J}_{103} \mathrm{Rh}^{-3}{ }_{\mathrm{P}}$ scalar coupling is observed as a 1:2:1 triplet with components lying along a negative diagonal. The ${ }^{103} \mathrm{Rh}-{ }^{1} \mathrm{H}$ coupling is absent in the directly detected ${ }^{1} \mathrm{H}$ dimension through ${ }^{103} \mathrm{Rh}$ decoupling during the data acquisition time. (b) Hydride ${ }^{1} \mathrm{H}$ NMR signal arising from the $1 \mathrm{D}{ }^{1} \mathrm{H}$ NMR spectrum of $\mathbf{I}$. (c) Hydride ${ }^{1} \mathrm{H}$ NMR signal arising from the $1 \mathrm{D}{ }^{1} \mathrm{H}$ NMR spectrum of $\mathbf{V}$. Details of the $2 \mathrm{D}\left[{ }^{1} \mathrm{H},{ }^{103} \mathrm{Rh}\right]$ data acquisition conditions are described in the experimental section of this Supporting Information. 


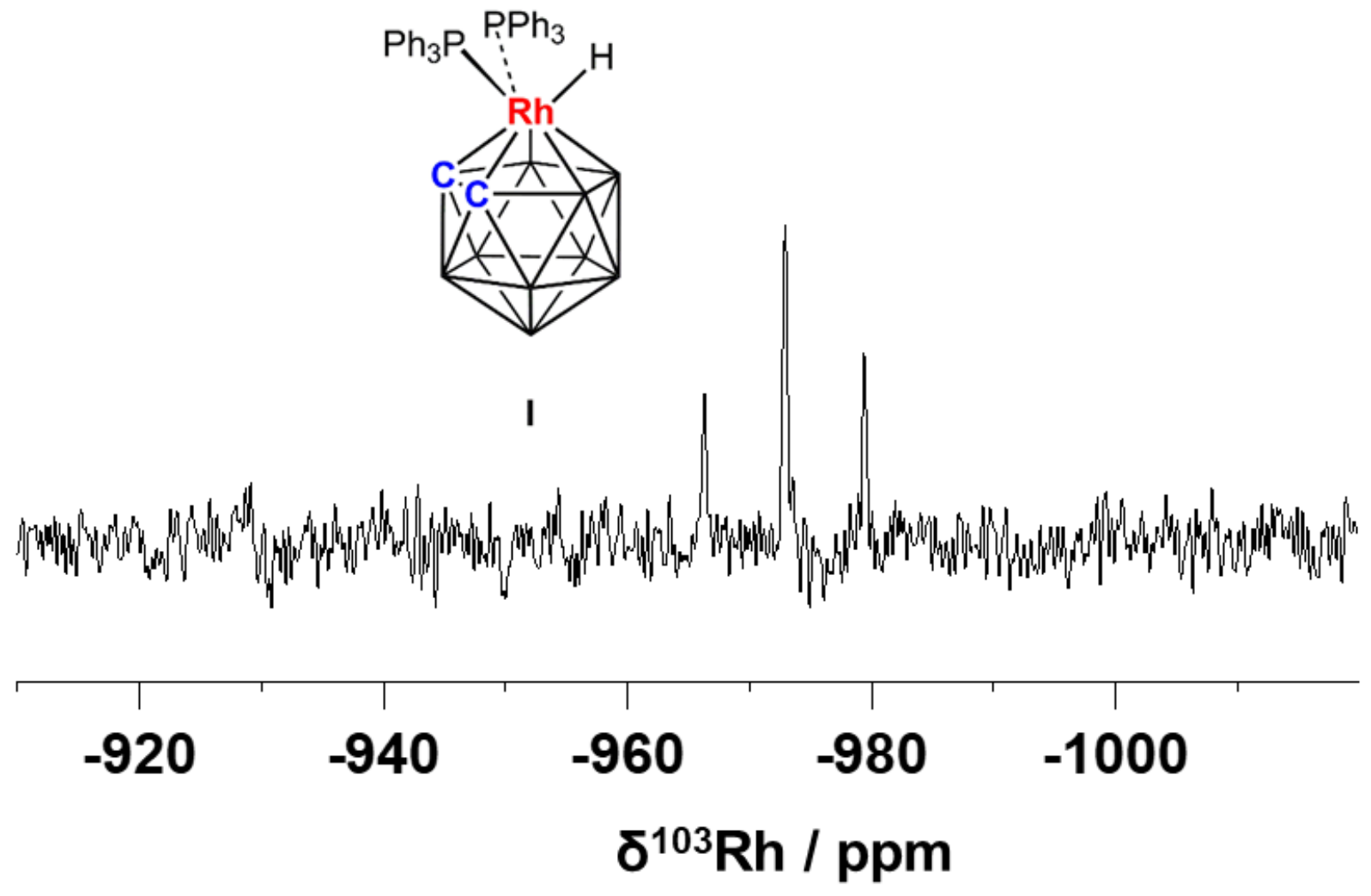

Figure S2. Directly observed 1D ${ }^{103} \mathrm{Rh}-\left\{{ }^{1} \mathrm{H}\right\}$-INEPT NMR spectrum for $\mathbf{I}$ acquired at a magnetic field of 14.1 $\mathrm{T}(600.130 \mathrm{MHz}$ for proton resonance) showing the expected 1:2:1 triplet pattern through the presence of ${ }^{1} \mathrm{~J}_{103} \mathrm{Rh}^{3}{ }^{3} \mathrm{P}$ scalar coupling.

\section{Experimental Section}

All NMR data were acquired on a three r.f. channel Bruker AVANCE $\mathrm{II}^{+}$NMR spectrometer equipped with a $14.1 \mathrm{~T}$ UltraShield $^{\mathrm{TM}}$ superconducting magnet housing a $5 \mathrm{~mm} \varnothing$ BBO-z-ATMA probehead capable of being manually tuned on the X-nucleus channel to resonance frequencies suitable for ${ }^{103} \mathrm{Rh}$ observation. The probehead temperature was maintained at $298 \mathrm{~K}$ for all samples measured. Samples were solubilized in $\mathrm{CDCl}_{3}$. Data were acquired and processed under Topspin 3.5 patch level 7 running on a Hewlett-Packard Z420 Workstation equipped with an Intel Xeon CPU E5-1620 v2 processor operating at $3.70 \mathrm{GHz}$ under Windows 7 Professional edition.

Calibration of approximate ${ }^{103} \mathrm{Rh}$ frequency offset. Since the ${ }^{103} \mathrm{Rh}$ resonance frequency was unknown for $\mathbf{I}$ and $\mathbf{V}$, the approximate frequency region in which the ${ }^{103} \mathrm{Rh}$ NMR response would appear was initially probed indirectly using the Bruker pulse program decp90, with proton as the observation nucleus. Parameters were set as follows: Proton offset, o1p $=-8.125 \mathrm{ppm}, \mathrm{sw}=5 \mathrm{ppm}, \mathrm{td}=8192, \mathrm{ds}=2, \mathrm{~ns}=4$, $\mathrm{aq}=1.36 \mathrm{~s}$. The indirect frequency was set to a basic value of $19.12 \mathrm{MHz} .{ }^{1} J_{\mathrm{HRh}}$ was set to a value of 17.5 $\mathrm{Hz}$, with relaxation delay, $\mathrm{d} 1=2.0$ and $\mathrm{ns}=4$. The initial value for the ${ }^{103} \mathrm{Rh}$ pulse power and pulse width 
were set to low values prior to full calibration. The ${ }^{103} \mathrm{Rh}$ frequency offset was varied whilst repeating the proton data acquisition until the smallest of differences was detected in the appearance of the hydride signal response. Once this had been found, the frequency offset was arrayed to find the frequency at which the maximum signal disturbance was detected. Once the frequency was set, the ${ }^{103} \mathrm{Rh}$ pulse power was increased and the pulse width modified until the pulse was correctly calibrated for $90^{\circ}$ excitation. A final ${ }^{103} \mathrm{Rh}$ pulse power of $125.89 \mathrm{~W}(-21.0 \mathrm{~dB})$ gave a ${ }^{103} \mathrm{Rh}$ pw90 $=30.0 \mu \mathrm{s}$.

Acquisition of 2D [ $\left[{ }^{1} \mathbf{H},{ }^{103} \mathbf{R h}\right]$ HMQC NMR spectra. The final $2 \mathrm{D}\left[{ }^{1} \mathrm{H},{ }^{103} \mathrm{Rh}\right] \mathrm{HMQC}$ NMR spectra were acquired using the basic Bruker pulse program hmqcph. NMR data were acquired into 1024 complex data points over an $\omega_{2}$ frequency width of $3001.2 \mathrm{~Hz}(5.00 \mathrm{ppm}$, aq $=170 \mathrm{~ms})$ centred at the hydride proton chemical shift for either sample. The offset frequency of the indirectly detected dimension was set to the value found during the initial decp90 calibration phase and adapted by turns until the position of the NMR signal was finalized. A total of 64 States-TPPI $t_{1}$ increments were acquired with 2 transients per increment over an $\omega_{1}$ frequency width of $947.28 \mathrm{~Hz}$ equivalent to $50 \mathrm{ppm}$ at a resonance frequency of $18.9456572 \mathrm{MHz} .{ }^{103} \mathrm{Rh}$ GARP decoupling was applied during the acquisition time with a pulse power of $5.0119 \mathrm{~W}(-7.0 \mathrm{~dB})$ yielding a decoupler 90 degree pulse length of $150 \mu$ s. Full data sets were acquire in a total acquisition time of 4 minutes. Repeat measurements were made by varying the $\omega_{1}$ frequency width and offset until it was clear that the signal position was stable (i.e. the absence of antialiasing in the $\omega_{1}$ dimension was apparent).

Acquisition of 1D ${ }^{103} \mathbf{R h}-\left\{{ }^{1} \mathbf{H}\right\}$ NMR spectra. 1D ${ }^{103} \mathrm{Rh}-\left\{{ }^{1} \mathrm{H}\right\}$ NMR spectra were acquired as proof of direct observation of the ${ }^{103} \mathrm{Rh}$ response using the Bruker pulse program ineptrd. NMR data were acquired into 1024 complex data points over an $\omega_{2}$ frequency width of $3001.2 \mathrm{~Hz}$ equivalent to a ${ }^{103} \mathrm{Rh}$ chemical shift range of $158.411 \mathrm{ppm}(\mathrm{aq}=170 \mathrm{~ms})$. The spectrometer frequency for observation, sfo1, was set to 18.9456572 MHz. The decoupler frequency was set to the hydride proton resonance in each case. The relaxation delay, d1, was set to $1.5 \mathrm{~s}$. Pulse lengths were set on the basis of the decp90 calibration values. Data were acquired with 1024 transient during a total acquisition period of 30 minutes. For $\mathrm{V}$, this did not result in the detection of an NMR response, owing to the lower quantity of material present, resulting in signal below the level of detection under these conditions.

Referencing of ${ }^{103}$ Rh NMR Spectra. Following data acquisition, the ${ }^{103} \mathrm{Rh}$ NMR data were referenced according to the IUPAC recommendations for a unified scale of referencing for all NMR spectra. ${ }^{1,2}$

(1) Harris, R. K.; Becker, E. D.; Cabral de Menezes, S. M.; Goodfellow, R.; Granger, P. NMR Nomenclature. Nuclear Spin Properties and Conventions for Chemical Shifts (IUPAC Recommendations 2001). Pure Appl. Chem. 2001, 73, 1795-1818.

(2) Harris, R. K.; Becker, E. D.; Cabral de Menezes, S. M.; Goodfellow, R.; Granger, P.; Hoffman, R. E.; Zilm, K. W. Further conventions for NMR shielding and chemical shifts (IUPAC Recommendations 2001). Pure Appl. Chem. 2008, 80, 59-84. 


\section{Part C Details of Catalytic Studies}

\section{Isomerisation of 1-hexene}

A J. Young NMR tube was flushed with $\mathrm{N}_{2}$ and charged with 1-hexene $(188 \mu \mathrm{L}, 1.503 \mathrm{mmol})$ and mesitylene (internal standard, $104 \mu \mathrm{L}, 0.748 \mathrm{mmol}) . \mathrm{A} \mathrm{CDCl}_{3}(1 \mathrm{~mL})$ solution of the catalyst $(0.003 \mathrm{mmol})$ was added and the progress of the reaction monitored by ${ }^{1} \mathrm{H}$ NMR spectroscopy. NMR yields were determined by the relative integrals of the products against the mesitylene internal standard (product chemical shifts used underlined, trans-3-hexene and cis-3-hexene could not be distinguished). Reactions completed in duplicate and average NMR yields quoted.

1-Hexene: ${ }^{1} \mathrm{H}$ NMR $\left(\mathrm{CDCl}_{3}\right)$; $\delta$ 5.79-5.69 $\left(1 \mathrm{H}, \mathrm{CH}_{2}=\mathrm{CH}\right), 5.01-4.93\left(2 \mathrm{H}, \mathrm{CH}_{2}=\mathrm{CH}\right), 1.99-1.94(2 \mathrm{H}$, $\mathrm{CH} 2), 1.32-1.21\left(4 \mathrm{H}, 2 \times \mathrm{CH}_{2}\right), 0.86-0.83\left(3 \mathrm{H}, \mathrm{CH}_{3}\right)$.

Trans-2-hexene: ${ }^{1} \mathrm{H}$ NMR $\left(\mathrm{CDCl}_{3}\right) ; \delta 5.41-5.37(2 \mathrm{H}, 2 \times \mathrm{CH}), 1.95-1.90\left(2 \mathrm{H}, \mathrm{CH}_{2}\right), \underline{1.60-1.59}\left(3 \mathrm{H}, \mathrm{CH}_{3}\right)$, 1.38-1.29 (2H, $\left.\mathrm{CH}_{2}\right), 0.88-0.84\left(3 \mathrm{H}, \mathrm{CH}_{3}\right)$.

Cis-2-hexene: ${ }^{1} \mathrm{H}$ NMR $\left(\mathrm{CDCl}_{3}\right) ; \delta$ 5.51-5.38 $(2 \mathrm{H}, 2 \times \mathrm{CH}), 2.01-1.95\left(2 \mathrm{H}, \mathrm{CH}_{2}\right), \underline{1.54-1.52}\left(3 \mathrm{H}, \mathrm{CH}_{3}\right)$, $1.38-1.28\left(2 \mathrm{H}, \mathrm{CH}_{2}\right), 0.89-0.85\left(3 \mathrm{H}, \mathrm{CH}_{3}\right)$.

Trans-3-hexene: ${ }^{1} \mathrm{H}$ NMR $\left(\mathrm{CDCl}_{3}\right) ; \delta \underline{5.43}(2 \mathrm{H}, 2 \times \mathrm{CH}), 2.00\left(4 \mathrm{H}, 2 \times \mathrm{CH}_{2}\right), 0.79\left(3 \mathrm{H}, 2 \times \mathrm{CH}_{3}\right)$.

Cis-3-hexene: ${ }^{1} \mathrm{H}$ NMR $\left(\mathrm{CDCl}_{3}\right) ; \delta \underline{5.33}(2 \mathrm{H}, 2 \times \mathrm{CH}), 2.03\left(4 \mathrm{H}, 2 \times \mathrm{CH}_{2}\right), 0.96\left(3 \mathrm{H}, 2 \times \mathrm{CH}_{3}\right)$.

Mesitylene: ${ }^{1} \mathrm{H}$ NMR $\left(\mathrm{CDCl}_{3}\right) ; \delta 6.91\left(3 \mathrm{H}, \mathrm{C}_{6} \mathrm{H}_{3}\right), 2.38\left(9 \mathrm{H}, 3 \times \mathrm{CH}_{3}\right)$.

\begin{tabular}{|c|c|c|c|c|c|}
\hline catalyst precursor & time (m) & trans-2-hexene (\%) & cis-2-hexene (\%) & 3-hexenes (\%) & total yield (\%) \\
\hline \multirow{4}{*}{ I } & 60 & 7.1 & 4.4 & 0 & 11.5 \\
\cline { 2 - 6 } & 120 & 12.1 & 7.5 & 0 & 19.6 \\
\cline { 2 - 6 } & 180 & 17.6 & 10.1 & 0 & 27.7 \\
\cline { 2 - 6 } & 240 & 22.0 & 11.9 & 0 & 33.9 \\
\cline { 2 - 6 } & 300 & 25.5 & 13.9 & 0 & 39.4 \\
\cline { 2 - 6 } & 360 & 29.2 & 15.4 & 0 & 44.6 \\
\hline \multirow{5}{*}{$\mathbf{V}$} & 5 & 7.8 & 5.2 & 0 & 13.0 \\
\cline { 2 - 6 } & 30 & 28.4 & 17.2 & 0 & 45.6 \\
\cline { 2 - 6 } & 60 & 46.0 & 24.9 & 0 & 70.9 \\
\cline { 2 - 6 } & 120 & 59.9 & 26.9 & 2.8 & 89.6 \\
\cline { 2 - 6 } & 180 & 63.4 & 26.8 & 6.2 & 96.4 \\
\cline { 2 - 6 } & 5 & 6.8 & 5.3 & 0 & 12.1 \\
\hline \multirow{5}{*}{$\mathbf{1}$} & 30 & 14.6 & 11.1 & 0 & 25.7 \\
\cline { 2 - 6 } & 60 & 24.2 & 17.2 & 0 & 41.4 \\
\hline & 120 & 39.1 & 25.6 & 0 & 64.7 \\
\cline { 2 - 6 } & 180 & 51.3 & 29.6 & 0 & 80.9 \\
\hline
\end{tabular}




\begin{tabular}{|c|c|c|c|c|c|}
\hline & 240 & 61.3 & 29.2 & 0 & 90.5 \\
\hline & 300 & 68.8 & 26.8 & 0 & 95.6 \\
\hline \multirow{3}{*}{$2 \alpha$} & 5 & 17.0 & 14.0 & 0 & 31.0 \\
\hline & 30 & 40.9 & 28.5 & 1.6 & 71.0 \\
\hline & 60 & 61.3 & 27.9 & 7.3 & 96.5 \\
\hline \multirow{3}{*}{$2 \beta$} & 5 & 19.1 & 15.1 & 0 & 34.2 \\
\hline & 30 & 42.3 & 28.8 & 2.2 & 73.3 \\
\hline & 60 & 61.0 & 27.2 & 8.5 & 96.7 \\
\hline \multirow{4}{*}{$3 \alpha$} & 5 & 7.7 & 6.4 & 0 & 14.1 \\
\hline & 30 & 31.0 & 22.0 & 0 & 53.0 \\
\hline & 60 & 52.3 & 31.0 & 1.0 & 84.3 \\
\hline & 90 & 62.3 & 28.4 & 5.8 & 96.5 \\
\hline \multirow{4}{*}{$3 \beta$} & 5 & 8.7 & 7.0 & 0 & 15.7 \\
\hline & 30 & 29.9 & 20.5 & 0 & 50.4 \\
\hline & 60 & 47.5 & 29.9 & 4.3 & 81.7 \\
\hline & 90 & 48.9 & 33.8 & 13.2 & 95.9 \\
\hline
\end{tabular}

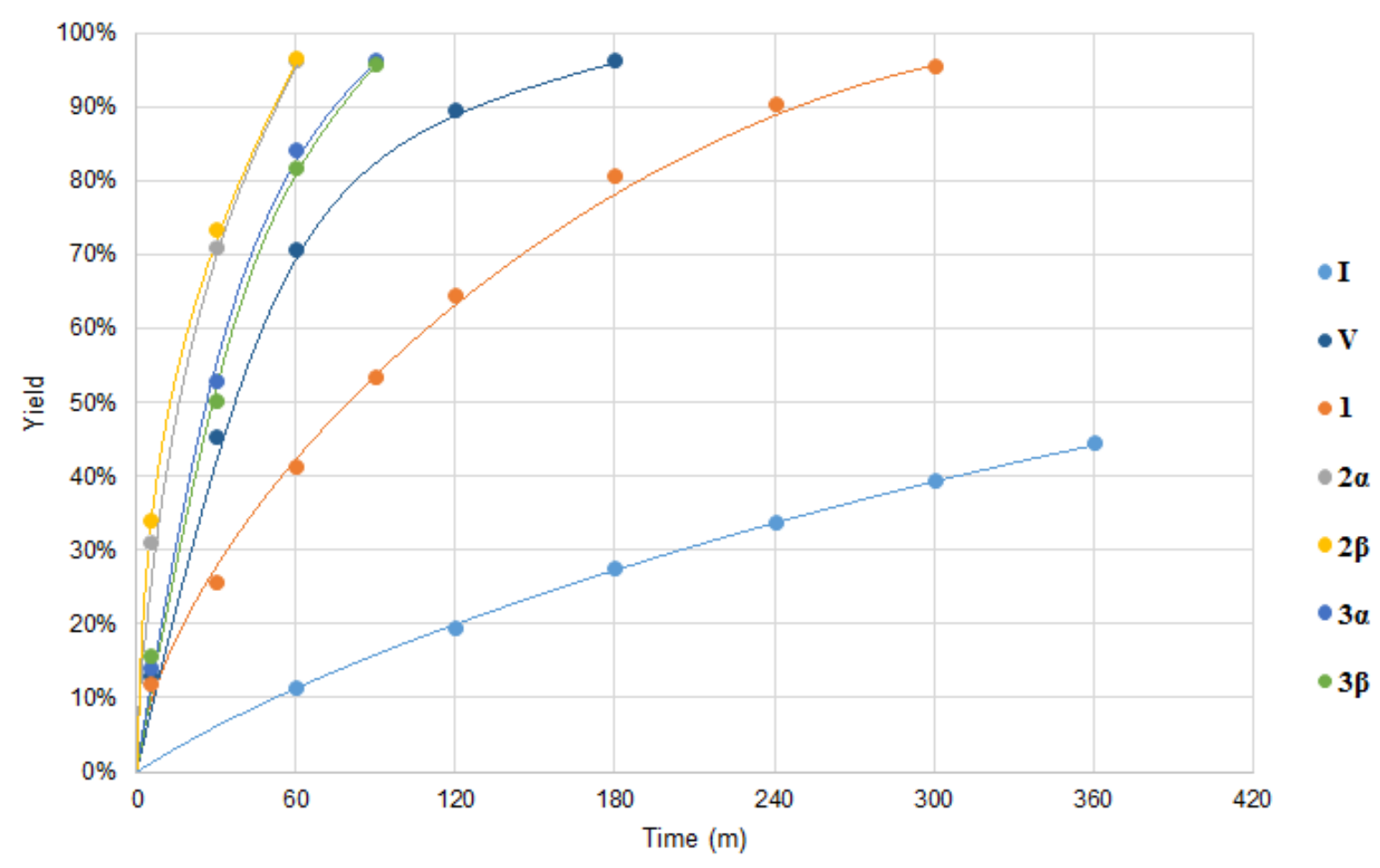




\section{Hydrosilylation of acetophenone}

Acetophenone $(37 \mu \mathrm{L}, 0.317 \mathrm{mmol})$, diphenylsilane $(74 \mu \mathrm{L}, 0.399 \mathrm{mmol})$ and mesitylene (internal standard, $22 \mu \mathrm{L}, 0.158 \mathrm{mmol}$ ) were combined in a J. Young NMR tube previously flushed with $\mathrm{N}_{2}$. A $\mathrm{CDCl}_{3}(1 \mathrm{~mL})$ solution of the catalyst precursor $(0.0016 \mathrm{mmol})$ was added, the reagents heated to $55^{\circ} \mathrm{C}$ and the progress of the reaction monitored by ${ }^{1} \mathrm{H}$ NMR spectroscopy. NMR yields were determined by the relative integrals of the products against the mesitylene internal standard (product chemical shifts used underlined). Reactions completed in duplicate and average NMR yields quoted.

Acetophenone: ${ }^{1} \mathrm{H}$ NMR $\left(\mathrm{CDCl}_{3}\right) ; \delta$ 7.96-7.94 $\left(2 \mathrm{H}, \mathrm{C}_{6} H_{5}\right), 7.57-7.55\left(1 \mathrm{H}, \mathrm{C}_{6} H_{5}\right), 7.47-7.43\left(2 \mathrm{H}, \mathrm{C}_{6} H_{5}\right)$, $2.59\left(3 \mathrm{H}, \mathrm{CH}_{3}\right)$.

Diphenylsilane: ${ }^{1} \mathrm{H}$ NMR $\left(\mathrm{CDCl}_{3}\right) ; \delta$ 7.65-7.64 (4H, $\left.\mathrm{C}_{6} H_{5}\right), 7.43-7.39\left(6 \mathrm{H}, \mathrm{C}_{6} H_{5}\right), 4.98(2 \mathrm{H}, 2 \times \mathrm{Si} H)$.

Diphenyl(1-phenylethoxy)silane: ${ }^{1} \mathrm{H}$ NMR $\left(\mathrm{CDCl}_{3}\right) ; \delta 7.83-7.32\left(15 \mathrm{H}, \mathrm{C}_{6} H_{5}\right), \underline{5.55}(1 \mathrm{H}, \mathrm{SiH}), 5.12(1 \mathrm{H}$, $\mathrm{CH}), \underline{1.62}\left(3 \mathrm{H}, \mathrm{CH}_{3}\right)$.

Diphenyl $\left\{(1-\right.$ phenylvinyl)oxy $\}$ silane: ${ }^{1} \mathrm{H}$ NMR $\left(\mathrm{CDCl}_{3}\right) ; \delta 7.83-7.32\left(15 \mathrm{H}, \mathrm{C}_{6} H_{5}\right), \underline{5.85}(1 \mathrm{H}, \mathrm{SiH}), \underline{5.05}$ $\left(1 \mathrm{H}, \mathrm{CH}_{2}\right), 4.65\left(1 \mathrm{H}, \mathrm{CH}_{2}\right)$.

Mesitylene: ${ }^{1} \mathrm{H}$ NMR $\left(\mathrm{CDCl}_{3}\right) ; \delta 6.91\left(3 \mathrm{H}, \mathrm{C}_{6} \mathrm{H}_{3}\right), 2.38\left(9 \mathrm{H}, 3 \times \mathrm{CH}_{3}\right)$.

\begin{tabular}{|c|c|c|c|c|}
\hline catalyst precursor & time $(\mathrm{m})$ & silyl ether (\%) & silyl enol ether (\%) & total yield (\%) \\
\hline \multirow{3}{*}{ I } & 60 & 17.7 & 23.9 & 41.6 \\
\hline & 120 & 23.2 & 65.5 & 88.7 \\
\hline & 1020 & 26.1 & 73.4 & 99.5 \\
\hline \multirow{2}{*}{$\mathbf{V}$} & 60 & 20.0 & 76.4 & 96.4 \\
\hline & 120 & 20.7 & 77.8 & 98.5 \\
\hline \multirow{3}{*}{1} & 60 & 9.9 & 22.3 & 32.3 \\
\hline & 120 & 14.0 & 29.0 & 43.1 \\
\hline & 1020 & 30.5 & 51.7 & 82.2 \\
\hline \multirow{3}{*}{$2 \alpha$} & 60 & 7.2 & 9.2 & 16.4 \\
\hline & 120 & 11.8 & 11.5 & 23.3 \\
\hline & 1020 & 35.6 & 29.8 & 65.4 \\
\hline \multirow{3}{*}{$2 \beta$} & 60 & 5.8 & 7.0 & 12.8 \\
\hline & 120 & 9.9 & 9.7 & 19.6 \\
\hline & 1020 & 36.0 & 29.4 & 65.4 \\
\hline \multirow{3}{*}{$3 \alpha$} & 60 & 8.1 & 11.3 & 19.4 \\
\hline & 120 & 11.9 & 16.5 & 28.4 \\
\hline & 1020 & 26.6 & 36.1 & 62.7 \\
\hline \multirow{2}{*}{$3 \beta$} & 60 & 9.5 & 13.8 & 23.3 \\
\hline & 120 & 13.3 & 17.9 & 31.2 \\
\hline
\end{tabular}




\begin{tabular}{|l|l|l|l|l|}
\hline & 1020 & 25.4 & 39.6 & 65.0 \\
\hline
\end{tabular}

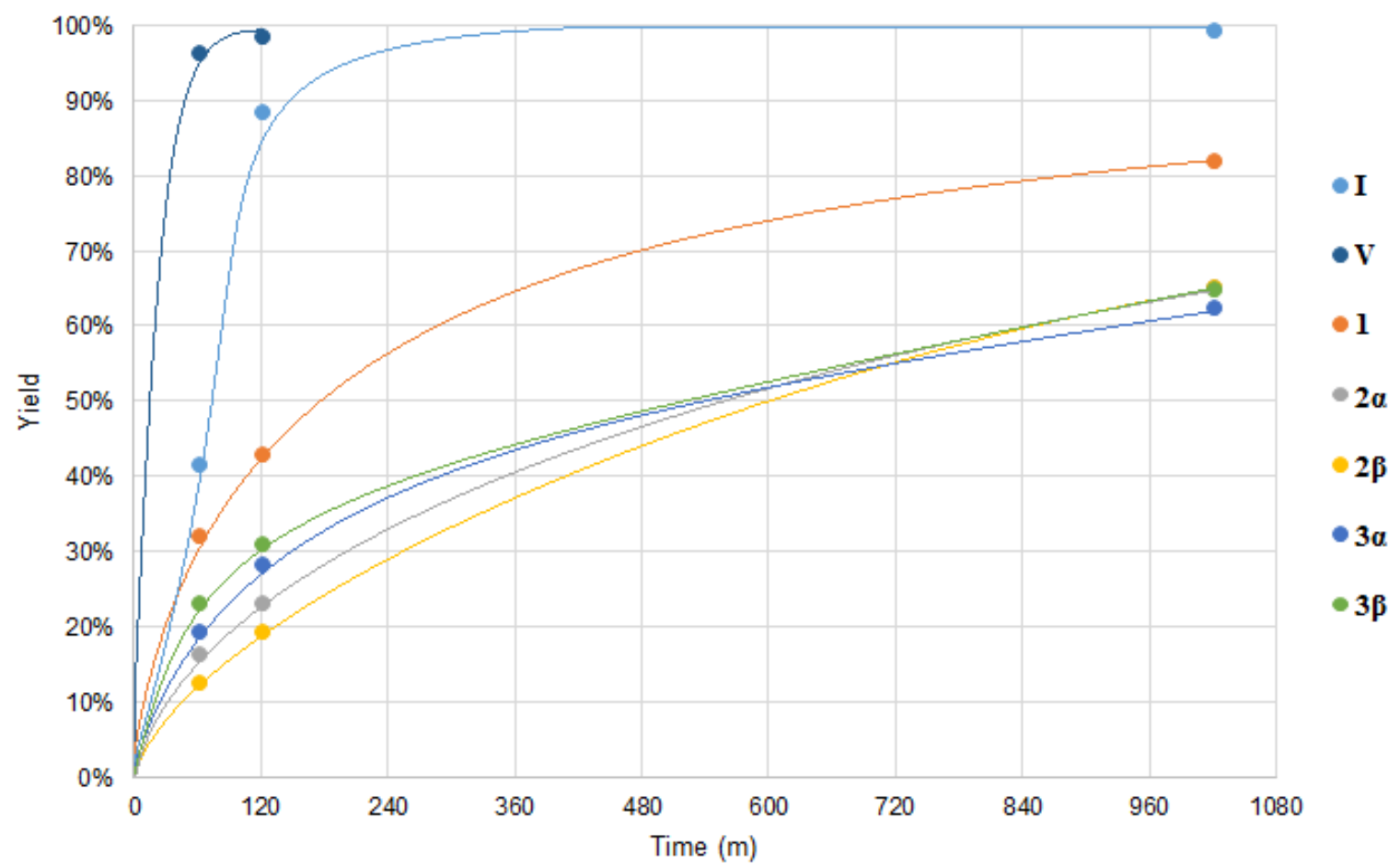

